\title{
Spin Excitations of a Proximate Kitaev Quantum Spin Liquid Realized in $\mathrm{Cu}_{2} \mathrm{IrO}_{3}$
}

\author{
Sean K. Takahashi, Jiaming Wang, Alexandre Arsenault, and Takashi Imai* \\ Department of Physics and Astronomy, McMaster University, Hamilton, Ontario, L8S 4M1, Canada \\ Mykola Abramchuk and Fazel Tafti \\ Department of Physics, Boston College, Chestnut Hill, Massachusetts 02467, USA \\ Philip M. Singer \\ Department of Chemical and Biomolecular Engineering, Rice University, \\ 6100 Main Street, Houston, Texas 77005-1892, USA
}

(Received 9 May 2019; revised manuscript received 22 July 2019; published 13 September 2019)

\begin{abstract}
Magnetic moments arranged at the corners of a honeycomb lattice are predicted to form a novel state of matter, the Kitaev quantum spin liquid, under the influence of frustration effects between bond-dependent Ising interactions. Some layered honeycomb iridates and related materials, such as $\mathrm{Na}_{2} \mathrm{IrO}_{3}$ and $\alpha-\mathrm{RuCl}_{3}$, are proximate to the Kitaev quantum spin liquid, but bosonic spin-wave excitations associated with undesirable antiferromagnetic long-range order mask the inherent properties of the Kitaev Hamiltonian. Here, we use ${ }^{63} \mathrm{Cu}$ nuclear quadrupole resonance to uncover the low-energy spin excitations in the nearly ideal honeycomb lattice of effective spin $S=1 / 2$ at the $\mathrm{Ir}^{4+}$ sites in $\mathrm{Cu}_{2} \mathrm{IrO}_{3}$. We demonstrate that, unlike $\mathrm{Na}_{2} \mathrm{IrO}_{3}$, Ir spin fluctuations exhibit no evidence for critical slowing-down toward magnetic long-range order in zero external magnetic field. Moreover, the low-energy spin excitation spectrum is dominated by a mode that has a large excitation gap comparable to the Ising interactions, a signature expected for Majorana fermions of the Kitaev quantum spin liquid.
\end{abstract}

DOI: 10.1103/PhysRevX.9.031047

Subject Areas: Condensed Matter Physics, Statistical Physics,

Strongly Correlated Materials

\section{INTRODUCTION}

Quantum spin liquids (QSLs) are a liquidlike state of matter, in which entangled spins remain paramagnetic without undergoing a magnetic long-range order. Identifying a QSL is the holy grail of quantum condensed matter research today [1-4]. A recent milestone in the search for QSLs is the rigorous theoretical solution of the Kitaev QSL for effective spin $S=1 / 2$ arranged in a honeycomb lattice [5]. The Kitaev Hamiltonian may be written as

$$
H_{K}=\sum_{\langle i, j\rangle} J_{K}^{\gamma} S_{i}^{\gamma} S_{j}^{\gamma}
$$

where $J_{K}^{\gamma}(\gamma=x, y$, and $z)$ represents the bond-dependent, frustrated Ising interaction as schematically shown in Fig. 1(a), and the lattice summation $\langle i, j\rangle$ is taken between

\footnotetext{
*imai@mcmaster.ca
}

Published by the American Physical Society under the terms of the Creative Commons Attribution 4.0 International license. Further distribution of this work must maintain attribution to the author(s) and the published article's title, journal citation, and DOI. nearest-neighbor spins. Kitaev proved based on analytic calculations that his Hamiltonian supports a quantum spin liquid ground state.

Besides possessing the ground state with no magnetic long-range order, the ideal Kitaev QSL is known to exhibit a variety of exotic properties [5,8-11]. For example, fractionalization of quantum spins leads to two types of Majorana fermions: itinerant Majorana fermions and localized fluxes. Therefore, elementary excitations in the Kitaev QSL are not conventional magnons (spin waves), and may exhibit an inherent gap in its spin excitation spectrum, depending on the anisotropy of the Ising interactions in Eq. (1). Thermodynamic properties could show nontrivial behavior, too. For example, the uniform spin susceptibility $\chi_{\text {spin }}$ is expected to increase or saturate with decreasing temperature, even if the excitation spectrum of Majorana fermions is inherently gapped [9-11]; this is due to the nonconservative nature of the $z$ component of the total $\operatorname{spin} S_{z}$.

The $\mathrm{Ir}^{4+}, \mathrm{Rh}^{4+}$, and $\mathrm{Ru}^{3+}$ ions in $\mathrm{Na}_{2} \mathrm{IrO}_{3}$ [12], $\mathrm{Li}_{2} \mathrm{RhO}_{3}$ [13], $\alpha-\mathrm{RuCl}_{3}$ [14], and more recently discovered $\mathrm{H}_{3} \mathrm{LiIr}_{2} \mathrm{O}_{6}$ [15] are under the influence of spin-orbit interactions, possess effective spin $S_{i}=1 / 2$, and interact with each 


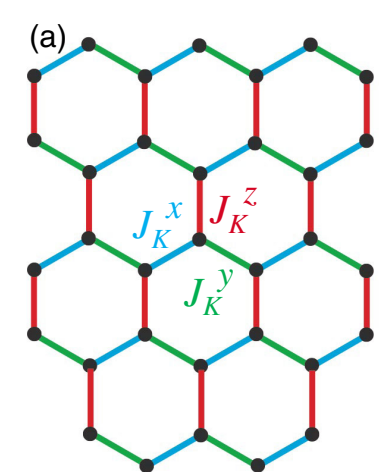

(b)

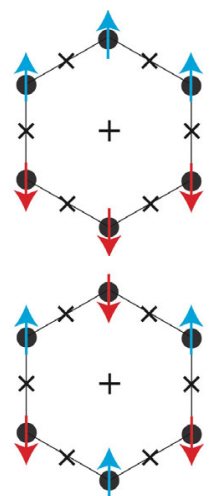

(d)

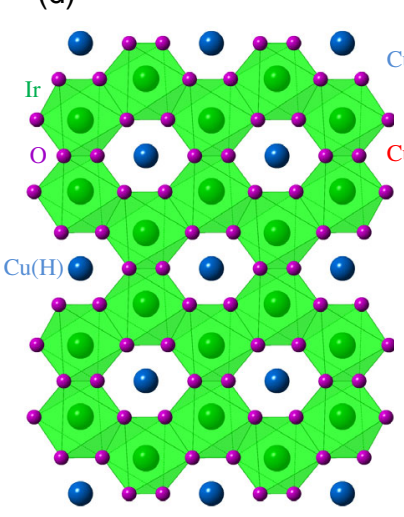

(e)

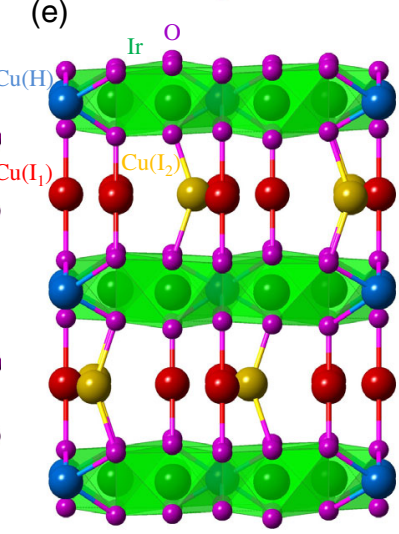

FIG. 1. (a) Kitaev lattice of effective spin $S=1 / 2$ (represented by bullets) at the corners of a honeycomb structure. Each spin interacts with three neighboring spins with Ising interactions $J_{K}^{x}$ (blue bonds), $J_{K}^{y}$ (green bonds), and $J_{K}^{z}$ (red bonds). The frustration effects between the bonds lead to formation of Kitaev quantum spin liquid. Possible long-range ordering patterns of Ir spins [6,7] in (b) zigzag and (c) Néel structure. + symbols show the location of the $\mathrm{Cu}(\mathrm{H})$ sites, while $\times$ symbols indicate the approximate location of $\mathrm{Cu}\left(\mathrm{I}_{1}\right)$ sites above or below the honeycomb planes. (d) Honeycomb lattice formed by $\mathrm{Ir}^{4+}$ ions in $\mathrm{Cu}_{2} \mathrm{IrO}_{3}$, viewed along the crystal $c^{*}$ axis. Each $\mathrm{Ir}^{4+}$ (green) is surrounded by six $\mathrm{O}^{2-}$ ions (purple) at the corners of an octahedron. $\mathrm{Cu}^{+}(\mathrm{H})$ (light blue) is at the center of each iridium hexagon. (e) Side view of the $\mathrm{Ir}^{4+}$ planes viewed along the crystal $a$ axis, with the interplanar $\mathrm{Cu}^{+}\left(\mathrm{I}_{1}\right)$ (red) and $\mathrm{Cu}^{+}\left(\mathrm{I}_{2}\right)$ (yellow) sites. O-Cu( $\left.\mathrm{I}_{1}\right)-\mathrm{O}$ bond is straight, whereas $\mathrm{O}-\mathrm{Cu}\left(\mathrm{I}_{2}\right)-\mathrm{O}$ bond is buckled.

other primarily via frustrated Ising interactions [16]. Therefore, the honeycomb planes formed by these spins have been proposed to harbor Kitaev QSL [16-18]. In reality, additional interactions, such as Heisenberg's exchange term, $H_{H}=\sum_{\langle i, j\rangle} J_{H} \mathbf{S}_{i} \cdot \mathbf{S}_{j}$, and the symmetric off-diagonal exchange term, $H_{\Gamma}=\sum_{\langle i, j\rangle} \Gamma\left(S_{i}^{\alpha} S_{j}^{\beta}+S_{i}^{\beta} S_{j}^{\alpha}\right)$ $[3,16,17]$, perturb the Kitaev Hamiltonian in Eq. (1), leading to the more complicated Kitaev-Heisenberg Hamiltonian:

$$
H_{K H}=H_{K}+H_{H}+H_{\Gamma}
$$

Theoretical calculations established that the KitaevHeisenberg Hamiltonian has a long-range ordered ground (a)

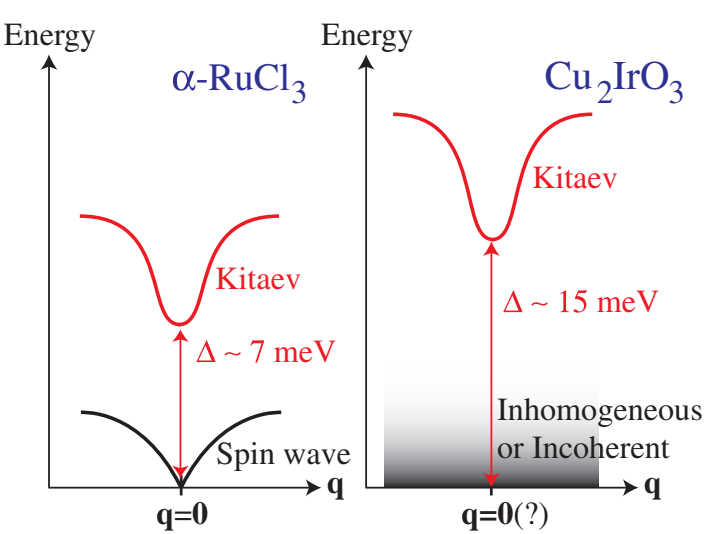

(b)

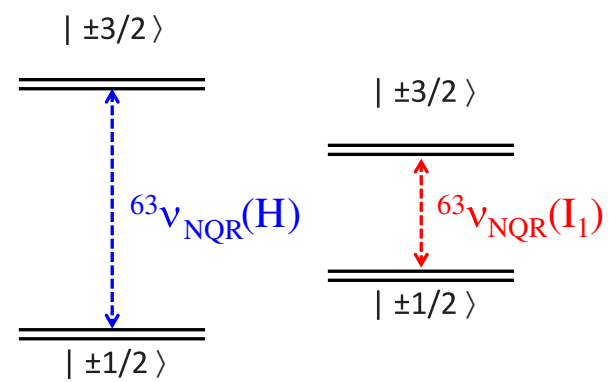

FIG. 2. (a) Conceptual diagrams of the spin excitation spectrum in Kitaev candidate material $\alpha-\mathrm{RuCl}_{3}$ with additional spin-wavelike modes below the gap $\Delta \sim 7 \mathrm{meV}$ [19-21] (left), and $\mathrm{Cu}_{2} \mathrm{IrO}_{3}$ with only spatially inhomogeneous, incoherent spin excitations at low energies (right). (b) Schematic diagram of the quadrupolar splitting between the nuclear spin $| \pm 1 / 2\rangle$ and $| \pm 3 / 2\rangle$ states for ${ }^{63} \mathrm{Cu}(\mathrm{H})$ and ${ }^{63} \mathrm{Cu}\left(\mathrm{I}_{1}\right)$ sites.

state rather than the spin liquid state in a broad parameter space [3]. Experimentally, $\mathrm{Na}_{2} \mathrm{IrO}_{3}$ [12], the first model material proposed for the Kitaev-Heisenberg model [17], indeed has a zigzag antiferromagnetic long-range order below $T_{N} \sim 17 \mathrm{~K}$, as shown schematically in Fig. 1(b) $[6,7]$. Such a long-range ordered ground state conceals the inherent properties of the Kitaev QSL. In particular, bosonic spin-wave excitations (with total spin 1) associated with the undesirable antiferromagnetic long-range order dominate the low-energy sector of the spin excitation spectrum in $\mathrm{Na}_{2} \mathrm{IrO}_{3}$ [7] and $\alpha-\mathrm{RuCl}_{3}$ [19-21], as shown in the left-hand panel of Fig. 2(a). This makes it difficult to identify the intrinsic excitations expected for Eq. (1), i.e., the fractionalized Majorana fermions (with total spin 1/2) and pairs of gauge fluxes (with total spin 1). Untangling the influence of $H_{H}$ and $H_{\Gamma}$ on $H_{K}$ has been the focus of intense research both theoretically and experimentally, but there are only a handful of proximate Kitaev materials known to date.

The recent discovery of $\mathrm{Cu}_{2} \mathrm{IrO}_{3}$ [22] by substitution of $\mathrm{Cu}^{+}$ions into the $\mathrm{Na}^{+}$sites of $\mathrm{Na}_{2} \mathrm{IrO}_{3}$ paved a new avenue to investigate the Kitaev QSL. We present the crystal structure of $\mathrm{Cu}_{2} \mathrm{IrO}_{3}$ in Figs. 1(d) and 1(e) [22]. Both the $\mathrm{Ir}-\mathrm{Ir}-\mathrm{Ir}\left(118.7^{\circ}-122.5^{\circ}\right)$ and $\mathrm{Ir}-\mathrm{O}-\mathrm{Ir}\left(95^{\circ}-98^{\circ}\right)$ bond angles are closer to the ideal Kitaev geometry of $120^{\circ}$ and 
$90^{\circ}[16,17]$, respectively, than those of $\mathrm{Na}_{2} \mathrm{IrO}_{3}\left(114.9^{\circ}-\right.$ $124.2^{\circ}$ and $98^{\circ}-99.4^{\circ}$, respectively [12]). There are two distinct types of $\mathrm{Cu}$ sites in $\mathrm{Cu}_{2} \mathrm{IrO}_{3}: \mathrm{Cu}(\mathrm{H})$ sites are located within the honeycomb plane in the middle of each hexagon formed by six Ir sites, while interplanar $\mathrm{Cu}(\mathrm{I})$ sites sit halfway between honeycomb planes [i.e., $\mathrm{Cu}(\mathrm{I})_{1.5} \mathrm{Cu}(\mathrm{H})_{0.5} \mathrm{IrO}_{3}$ ]. The uniform magnetic susceptibility $\chi$ observed for $\mathrm{Cu}_{2} \mathrm{IrO}_{3}$ is nearly identical to that of $\mathrm{Na}_{2} \mathrm{IrO}_{3}$ except below $\sim 30 \mathrm{~K}$, where defect spins dominate $\chi$ in $\mathrm{Cu}_{2} \mathrm{IrO}_{3}[22,23]$. In the case of $\mathrm{Na}_{2} \mathrm{IrO}_{3}$, the theoretical fit of $\chi$ based on the Kitaev-Heisenberg Hamiltonian was very good, and led to the initial estimate of $J_{K} \sim 21 \mathrm{meV}$ and $J_{H} \sim-4 \mathrm{meV}$ [17]. In order to account for the zigzag ordered ground state, however, the additional off-diagonal exchange terms in Eq. (2) are required [3]. Subsequent firstprinciples calculations based on exact diagonalization, the quantum chemistry method, and perturbation theory led to $J_{K}=-16.8 \sim-29.4 \mathrm{meV}, J_{H}=0.5-3.2 \mathrm{meV}$, and $\Gamma \sim$ $1 \mathrm{meV}$ [24]. In view of the nearly identical $\chi$, we expect that spin-spin interactions in $\mathrm{Cu}_{2} \mathrm{IrO}_{3}$ are comparable to these estimations.

Unlike $\mathrm{Na}_{2} \mathrm{IrO}_{3}$, however, $\mathrm{Cu}_{2} \mathrm{IrO}_{3}$ shows no evidence for antiferromagnetic long-range order [22], and recent $\mu \mathrm{SR}[23,25]$ as well as our nuclear quadrupole resonance (NQR) measurements presented below establish that only $\sim 50 \%$ of the sample volume exhibits spin freezing below the onset temperature of $T_{f} \sim 10 \mathrm{~K}$. Moreover, muons exhibit no spin precession about static hyperfine field, and Ir spins remain dynamic even below $1 \mathrm{~K}[23,25]$. This means that the low-energy sector of the spin excitation spectrum in $\mathrm{Cu}_{2} \mathrm{IrO}_{3}$ is largely immune from the spin waves, as schematically shown in the right-hand panel of Fig. 2(a), and opens a wide temperature window above $T_{f}$ to probe the inherent properties of the Kitaev QSL.

On the other hand, $\mathrm{Cu}_{2} \mathrm{IrO}_{3}$ has its own complications arising from stacking faults, as is often the case for previously identified Kitaev candidate materials, and from the aforementioned defect spins [25]. The defect-induced enhanced susceptibility below $\sim 30 \mathrm{~K}$ is known to obey a peculiar scaling law [23], reminiscent of analogous scaling behavior reported earlier for other spin liquid candidate materials with disorder [26]. The origin and nature of the defect spins in $\mathrm{Cu}_{2} \mathrm{IrO}_{3}$ as well as in other related iridates [15,27-29] are a matter of intense research and are yet to be clarified. In the case of $\mathrm{Cu}_{2} \mathrm{IrO}_{3}$, x-ray absorption nearedge spectroscopy (XANES) measurements showed that up to $\sim 1 / 3$ of ${ }^{63} \mathrm{Cu}(\mathrm{H})$ sites are occupied by $\mathrm{Cu}^{2+}$ ions rather than $\mathrm{Cu}^{+}$ions [25]. To maintain charge neutrality, the charge state of an $\mathrm{Ir}^{4+}$ ion(s) in their vicinity may change, possibly to $\mathrm{Ir}^{3+}$ with null spin. If randomly distributed, they might create bond disorder [30] and/or site dilution in the Kitaev Hamiltonian, in addition to the decoration of the Kitaev lattice with additional spin $S=1 / 2$ at the $\mathrm{Cu}^{2+}$ sites. Curiously, the bulk susceptibility of $\mathrm{Cu}_{2} \mathrm{IrO}_{3}$ and $\mathrm{Na}_{2} \mathrm{IrO}_{3}$ shares nearly identical Weiss temperature, $\Theta_{w}=-110 \sim-123 \mathrm{~K}$ [22], whereas $\mathrm{Mg}^{2+}$ substitution into $\mathrm{Na}_{2} \mathrm{IrO}_{3}$ is known to suppress $\Theta_{w}$ quickly to almost zero [29].

In this paper, we use ${ }^{63} \mathrm{Cu} \mathrm{NQR}$, a variant of NMR, to probe intrinsic spin excitations in the paramagnetic state of Ir honeycomb layers in $\mathrm{Cu}_{2} \mathrm{IrO}_{3}$. A major thrust of our work is that we can probe the Ir honeycomb lattice using NQR in zero magnetic field, owing to the strong nuclear quadrupole interaction at the $\mathrm{Cu}$ sites. Moreover, the magnetic hyperfine coupling $A_{\mathrm{hf}}$ between $\mathrm{Cu}$ nuclear spins and Ir electron spins via the transferred spin polarization into the empty $\mathrm{Cu} 4 s$ orbital [31] is generally strong. Accordingly, defect spins do not overwhelm the intrinsic ${ }^{63} \mathrm{Cu}$ NQR and NMR properties of $\mathrm{Cu}_{2} \mathrm{IrO}_{3}$, unlike ${ }^{1} \mathrm{H}$ NMR data for the kagome lattice in herbertsmithite $\mathrm{ZnCu}_{3}(\mathrm{OH})_{6} \mathrm{Cl}_{2}$ [32-34] and for the honeycomb lattice of $\mathrm{H}_{3} \mathrm{LiIr}_{2} \mathrm{O}_{6}$ [15]. We demonstrate that $\mathrm{Cu}_{2} \mathrm{IrO}_{3}$ remains paramagnetic and exhibits a large gap $\Delta$ comparable to the magnitude of Ising interaction, $\Delta \sim 15 \mathrm{meV}\left(=0.5\left|J_{K}\right| \sim\right.$ $\left.0.7\left|J_{K}\right|\right)$ in the primary channel of spin excitations in zero external magnetic field, $B_{\text {ext }}=0$.

\section{EXPERIMENTAL METHODS}

We synthesized the powder samples of $\mathrm{Na}_{2} \mathrm{IrO}_{3}$, $\mathrm{Cu}_{2} \mathrm{IrO}_{3}$, and $\mathrm{Cu}_{1.5} \mathrm{Na}_{0.5} \mathrm{SnO}_{3}$ for NMR measurements via solid-state reaction and ion exchange as described in Refs. $[22,35]$. We conducted all NMR and NQR measurements using home-built NMR spectrometers with the standard $\pi / 2-\pi$ spin echo pulse sequence. The typical pulse width for the $\pi / 2$ pulse was $2-7 \mu \mathrm{s}$, and the pulse separation time was $\tau \sim 10 \mu \mathrm{s}$. We measured $1 / T_{1}$ using the inversion recovery method, whereby we applied a $\pi$ pulse, followed by a spin echo sequence for various delay times $t$. We fitted the inversion recovery of nuclear magnetization $M(t)$ to the exponential function expected for the observed transition [36,37], as discussed in detail in the Appendix A. We measured the NQR intensity near and below $T_{f}$ at the peak of the $\mathrm{Cu}\left(\mathrm{I}_{1}\right)$ and $\mathrm{Cu}(\mathrm{H})$ sites using a fixed delay time $\tau=10 \mu \mathrm{s}$. We uniaxially aligned the powder sample of $\mathrm{Cu}_{2} \mathrm{IrO}_{3}$ in Stycast 1266 [38] by curing the mixture for $12 \mathrm{~h}$ in a mold made of Teflon in a magnetic field of $9 \mathrm{~T}$.

\section{NMR RESULTS}

\section{A. ${ }^{63,65} \mathrm{Cu}$ NQR line shapes}

Naturally occurring ${ }^{63,65} \mathrm{Cu}$ isotopes at $\mathrm{Cu}(\mathrm{H})$ and $\mathrm{Cu}(\mathrm{I})$ sites have nuclear spin $I=3 / 2$ accompanied by a nuclear quadrupole moment ${ }^{63,65} \mathrm{Q}$, and the latter interacts with the electric field gradient (EFG) generated by electrons and ions in the lattice. As shown schematically in Fig. 2(b), this nuclear quadrupole interaction lifts the degeneracy of the nuclear spin $| \pm 1 / 2\rangle$ and $| \pm 3 / 2\rangle$ states even in the absence of an external magnetic field $B_{\text {ext }}$. It is therefore possible to conduct ${ }^{63,65} \mathrm{Cu}$ NMR based on the NQR techniques in $B_{\text {ext }}=0$ without perturbing the Ir electron spins with 
Zeeman effects, a crucial advantage of $\mathrm{Cu}_{2} \mathrm{IrO}_{3}$ for NMR investigation into the inherent Kitaev physics.

In Figs. 3(a) and 3(b), we compare the ${ }^{63,65} \mathrm{Cu}$ NQR line shapes observed for $\mathrm{Cu}_{2} \mathrm{IrO}_{3}$ and a nonmagnetic reference material $\mathrm{Cu}_{1.5} \mathrm{Na}_{0.5} \mathrm{SnO}_{3}$ [35] with $\mathrm{Na}_{0.5} \mathrm{Sn}$ honeycomb layers. The NQR peak frequency ${ }^{63,65} \nu_{\mathrm{NQR}}$ of the ${ }^{63,65} \mathrm{Cu}$ isotopes is proportional to ${ }^{63,65} Q$, where ${ }^{65} Q /{ }^{63} Q=0.927$, and hence each ${ }^{63} \mathrm{Cu} \mathrm{NQR}$ peak observed at ${ }^{63} \nu_{\mathrm{NQR}}$ is accompanied by a smaller ${ }^{65} \mathrm{Cu} \mathrm{NQR}$ peak at a lower frequency, ${ }^{65} \nu_{\mathrm{NQR}}=0.927 \times{ }^{63} \nu_{\mathrm{NQR}}$ with the intensity ratio set by their natural abundance, $69 \%$ and $31 \%$. The two pairs of similar ${ }^{63,65} \mathrm{Cu}$ NQR peaks observed below $30 \mathrm{MHz}$ for both $\mathrm{Cu}_{2} \mathrm{IrO}_{3}$ and $\mathrm{Cu}_{1.5} \mathrm{Na}_{0.5} \mathrm{SnO}_{3}$ indicate that they arise from two distinct types of the interlayer $\mathrm{Cu}\left(\mathrm{I}_{1}\right)$ and $\mathrm{Cu}\left(\mathrm{I}_{2}\right)$ sites [the $\mathrm{O}-\mathrm{Cu}\left(\mathrm{I}_{2}\right)-\mathrm{O}$ bond has a kink; see Fig. 1(e)]. We assign the remaining ${ }^{63,65} \mathrm{Cu} \mathrm{NQR}$ peaks observed at around $52 \mathrm{MHz}$ to the honeycomb $\mathrm{Cu}(\mathrm{H})$ sites.

We note that the Kitaev candidate materials tend to suffer from stacking faults. In the case of $\mathrm{Cu}_{2} \mathrm{IrO}_{3}$, due to the weak $\mathrm{Cu}-\mathrm{O}-\mathrm{Cu}$ bonds between the layers of $\mathrm{Cu}_{2} \mathrm{IrO}_{3}$, the material is prone to stacking faults in the form of twinning between the adjacent layers [35]. The stacking faults are
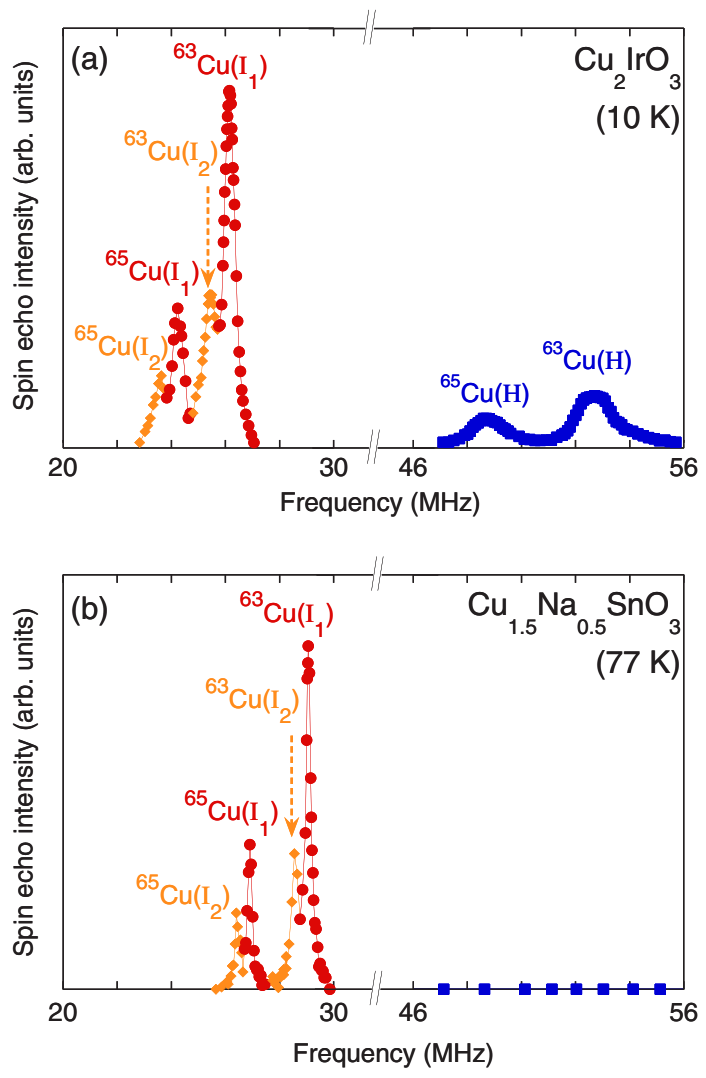

FIG. 3. (a) ${ }^{63,65} \mathrm{Cu}$ NQR line shape observed at $10 \mathrm{~K}$ for the inplane $\mathrm{Cu}(\mathrm{H})$ honeycomb site and the interplanar $\mathrm{Cu}\left(\mathrm{I}_{1}\right)$ and $\mathrm{Cu}\left(\mathrm{I}_{2}\right)$ sites of $\mathrm{Cu}_{2} \mathrm{IrO}_{3}$. (b) ${ }^{63,65} \mathrm{Cu} \mathrm{NQR}$ line shape observed at $77 \mathrm{~K}$ for nonmagnetic reference sample $\mathrm{Cu}_{1.5} \mathrm{Na}_{0.5} \mathrm{SnO}_{3}$, which lacks $\mathrm{Cu}(\mathrm{H})$ sites. extended disorders that happen over several unit cells; however, we have to effectively model them in one unit cell when we solve the crystal structure by Rietveld refinements. The result is two inequivalent dumbbell bonds, one straight and one twisted. If both bonds were perfectly straight, there would have been no twisting between adjacent layers and no stacking disorder. Our observation of two distinct types of dumbbell $\mathrm{Cu}\left(\mathrm{I}_{1}\right)$ and $\mathrm{Cu}\left(\mathrm{I}_{2}\right)$ sites supports this.

We summarize the temperature dependence of ${ }^{63} \mathrm{Cu}$ NQR line shapes in Figs. 4(a) and 4(b). We observed no splitting or broadening of the NQR line shapes due to static hyperfine magnetic fields arising from ordered magnetic moments, a typical NMR signature of antiferromagnetic long-range order (see Appendix $\mathrm{C}$ for magnetic NMR line broadening observed for $\mathrm{Na}_{2} \mathrm{IrO}_{3}$ below $T_{N}$ ). The NQR frequency ${ }^{63} \nu_{\mathrm{NQR}}$ summarized in Fig. 4(c) reflects the local lattice and charge environment. ${ }^{63} \mathrm{\nu}_{\mathrm{NQR}}$ at ${ }^{63} \mathrm{Cu}\left(\mathrm{I}_{1,2}\right)$ decreases smoothly toward higher temperatures due to thermal expansion, without exhibiting evidence for a
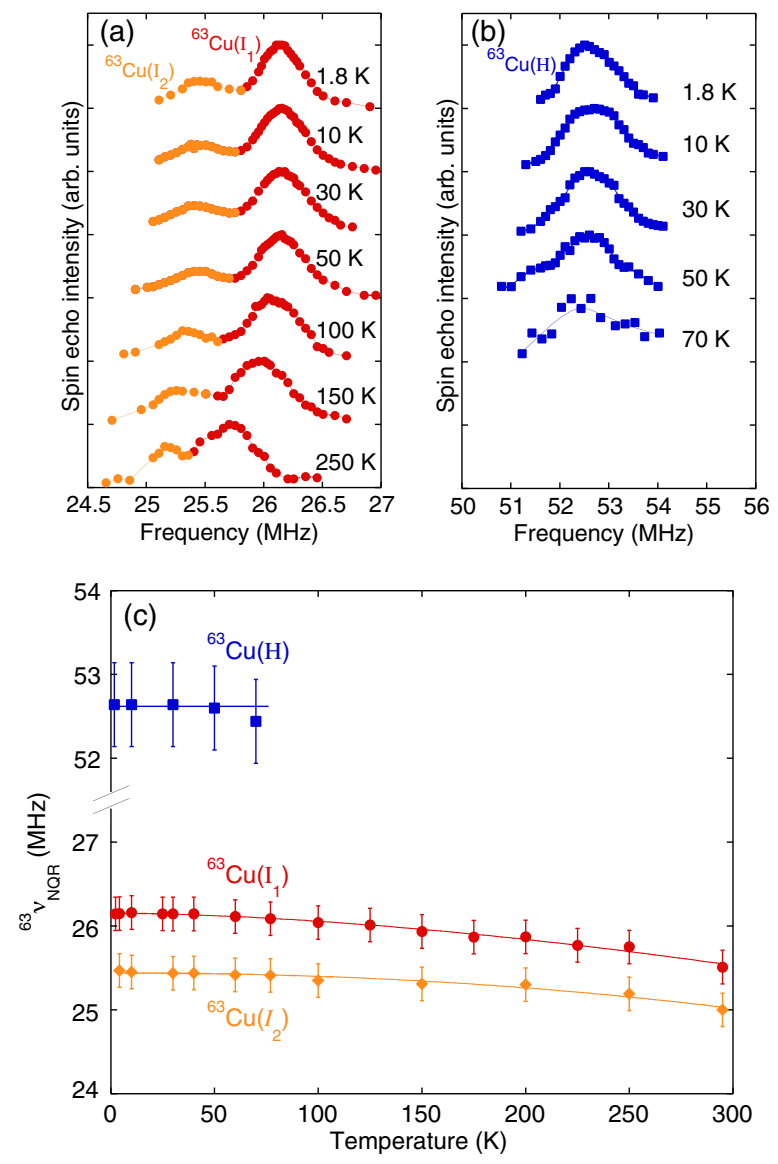

FIG. 4. (a),(b) ${ }^{63} \mathrm{Cu}$ NQR peaks observed for the ${ }^{63} \mathrm{Cu}\left(\mathrm{I}_{1,2}\right)$ and ${ }^{63} \mathrm{Cu}(\mathrm{H})$ sites at selected temperatures. For clarity, the origin of the vertical axis is shifted at different temperatures, and the peak intensity is normalized to 1 . (c) Temperature dependence of ${ }^{63} \nu_{\mathrm{NQR}}$. All solid lines are a guide for the eyes. 
structural transition or dimerization. We were unable to keep track of the ${ }^{63} \mathrm{Cu}(\mathrm{H}) \mathrm{NQR}$ signals above $\sim 70 \mathrm{~K}$ due to extremely weak signal intensity caused by very fast NMR relaxation rates. This is simply because the hyperfine coupling between ${ }^{63} \mathrm{Cu}(\mathrm{H})$ nuclear spins with six nearest-neighbor Ir spins is stronger than that at the ${ }^{63} \mathrm{Cu}\left(\mathrm{I}_{1,2}\right)$ sites, as discussed in detail in Sec. III E. Generally, detection of the ${ }^{63} \mathrm{Cu}$ NQR and NMR spin echo signals becomes difficult when the NMR spin-lattice relaxation rate $1 / T_{1}$ reaches $\sim 10^{4} \mathrm{sec}^{-1}$ [32].

Although there is no evidence for magnetic long-range order, we found that the integrated intensity of the ${ }^{63} \mathrm{Cu}$ NQR signals begins to decrease gradually below $T_{f} \sim 10 \mathrm{~K}$. This indicates that NMR relaxation rates become very fast in some domains. In Fig. 5(a), we summarize the temperature dependence of the lost fraction $f_{\mathrm{NQR}}$ of the NQR spin echo intensity, measured at a constant pulse separation time $\tau=10 \mu \mathrm{s}$, down to $1.5 \mathrm{~K} . f_{\mathrm{NQR}}$ shows qualitatively the same behavior as the volume fraction $f_{\mu \mathrm{SR}}$ estimated by $\mu \mathrm{SR}$ experiments [25], in which muons exhibit fast depolarization due to slow spin fluctuations in their vicinity $[23,25]$. Note that $f_{\mu \mathrm{SR}}$ saturates at $50 \%$ below $1 \mathrm{~K}$. $\left(f_{\mathrm{NQR}}\right.$ overestimates the volume fraction with slowed spin fluctuations, because the distribution of the spin-spin relaxation time $T_{2}$ prevented us from accurately taking into account the contributions of nuclear spins with fast $T_{2}$.) Combined with the fact that we found no evidence for divergent NMR relaxation rates for the observable NQR signals, we conclude that the loss of the ${ }^{63} \mathrm{Cu}$ NQR signals is primarily due to slowing of defect spin fluctuations below $T_{f}$. Since the ${ }^{63} \mathrm{Cu}$ NQR line shape for observable signals does not broaden even below $T_{f}$, the defect spins may be spatially confined in some domains, perhaps in the vicinity of stacking faults [25], rather than uniformly distributed throughout the entire $\mathrm{Ir}^{4+}$ honeycomb planes.

\section{B. Low-energy Ir spin excitations in $\boldsymbol{B}_{\text {ext }}=\mathbf{0}$}

We now turn our attention to our central results on lowfrequency Ir spin dynamics probed by the ${ }^{63} \mathrm{Cu}$ nuclear spin-lattice relaxation rate, $1 / T_{1}$. Quite generally, $1 / T_{1}$ probes the low-frequency component at the NQR frequency $\nu_{\mathrm{NQR}}$ of the fluctuating hyperfine magnetic field $h_{\text {perp }}=A_{\mathrm{hf}} S$ arising from the $\operatorname{Ir}$ spin $S$, i.e., $1 / T_{1}=\left(\gamma_{n}^{2} / 2\right) \int\left\langle h_{\text {perp }}(t) h_{\text {perp }}(0)\right\rangle \exp \left(-2 \pi i \nu_{\mathrm{NQR}} t\right) d t$, where $\gamma_{n} / 2 \pi=11.285 \mathrm{MHz} / \mathrm{T}$ is the ${ }^{63} \mathrm{Cu}$ nuclear gyromagnetic ratio, and perp indicates the component orthogonal to the quantization axis of the observed nuclear spins. The quantization axis of ${ }^{63} \mathrm{Cu}$ nuclear spins is along the $c$ axis for our NQR measurements, and hence our NQR $1 / T_{1}$ results probe the slow component at ${ }^{63} \nu_{\mathrm{NQR}}$ of Ir spin fluctuations within the $a b$ plane.
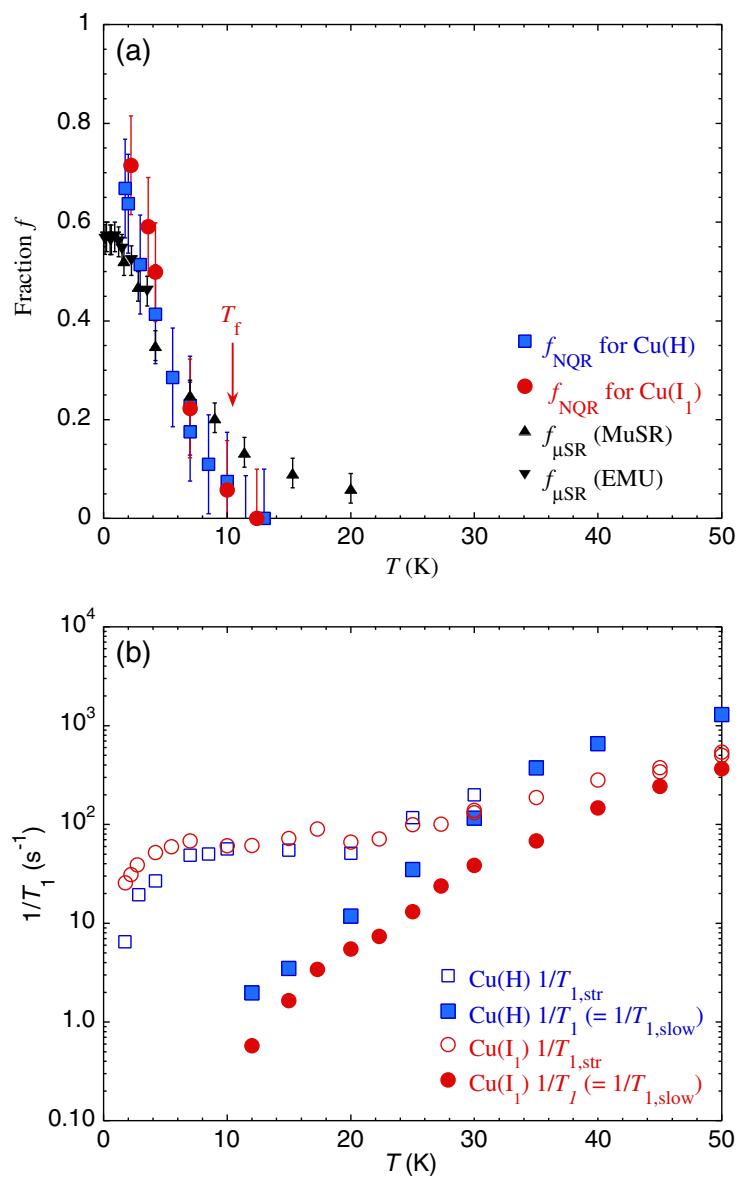

FIG. 5. (a) The lost fraction $f_{\mathrm{NQR}}$ of the ${ }^{63} \mathrm{Cu}$ NQR signal intensity measured at the ${ }^{63} \mathrm{Cu}\left(\mathrm{I}_{1}\right)$ and ${ }^{63} \mathrm{Cu}(\mathrm{H})$ sites at a fixed delay time $\tau=10 \mu \mathrm{s}$. Also plotted are the fraction $f_{\mu \mathrm{SR}}$ of the fast depolarizing component of the $\mu \mathrm{SR}$ signals, as measured by zero field $\mu \mathrm{SR}$ techniques using the MuSR (triangles) and EMU (downward triangles) spectrometers at the Rutherford Appleton Laboratory [25]. (b) A precursor of the spin freezing observed above $T_{f}$ in the spin-lattice relaxation rate $1 / T_{1}$. Intrinsic $1 / T_{1}$, obtained as the slower component $1 / T_{1 \text {,slow }}$ from the twocomponent fit with Eq. (A3) below $\sim 60 \mathrm{~K}$ (i.e., $1 / T_{1}=$ $1 / T_{1 \text {,slow }}$ below $\sim 60 \mathrm{~K}$ ), continues to slow down at low temperatures. In contrast, the sample averaged behavior represented by $1 / T_{1, \text { str }}$, estimated from the stretched fit of $M(t)$ with Eq. (A2), levels off below $\sim 30 \mathrm{~K}$. See Appendix A for details of the fit.

One can also write $1 / T_{1} \propto \Sigma_{\mathbf{q}}\left|A_{\mathrm{hf}}(\mathbf{q})\right|^{2} S\left(\mathbf{q}, E_{n}\right)$, where $A_{\mathrm{hf}}(\mathbf{q})$ is the wave-vector q-dependent hyperfine form factor [39]. $S\left(\mathbf{q}, E_{n}\right)$ is the dynamic spin structure factor of Ir spins at the very small excitation energy of $E_{n}=$ $h^{63} \nu_{\mathrm{NQR}} \sim 0.2 \mu \mathrm{eV}$, corresponding to the photon energy at the NQR frequency. $1 / T_{1}$ therefore probes the low-energy sector of the q-integrated spin excitation spectrum $S\left(\mathbf{q}, E_{n}\right)$ at energy $E_{n}$, weighted by $\left|A_{\mathrm{hf}}(\mathbf{q})\right|^{2}$.

In Fig. 6(a), we summarize $1 / T_{1}$ at the ${ }^{63} \mathrm{Cu}(\mathrm{H})$, ${ }^{63} \mathrm{Cu}\left(\mathrm{I}_{1}\right)$, and ${ }^{63} \mathrm{Cu}\left(\mathrm{I}_{2}\right)$ sites measured at the peak frequency of the NQR line shapes. The hyperfine coupling $A_{\mathrm{hf}}$ of Ir spins with the ${ }^{63} \mathrm{Cu}(\mathrm{H})$ nuclear spins is stronger than with 

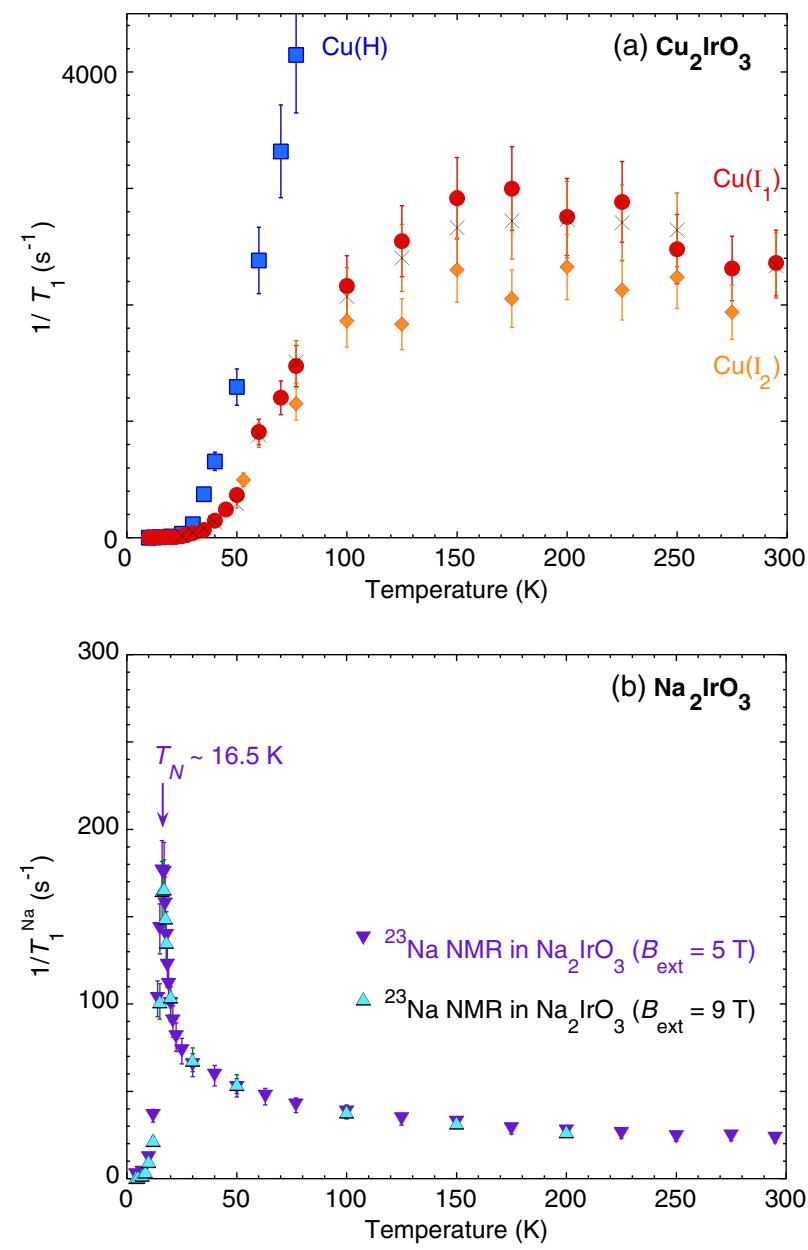

FIG. 6. (a) Filled symbols: intrinsic $1 / T_{1}$ measured in $B_{\text {ext }}=0$ with $\mathrm{NQR}$ at ${ }^{63} \mathrm{Cu}\left(\mathrm{I}_{1}\right),{ }^{63} \mathrm{Cu}\left(\mathrm{I}_{2}\right)$, and ${ }^{63} \mathrm{Cu}(\mathrm{H})$ sites of $\mathrm{Cu}_{2} \mathrm{IrO}_{3}$. Note that $1 / T_{1}$ plotted below $\sim 60 \mathrm{~K}$ is the intrinsic, slow component estimated from the two-component fit with Eq. (A3) (i.e., $1 / T_{1}=1 / T_{1 \text {,slow }}$ below $\sim 60 \mathrm{~K}$ ). Also plotted with $\times$ symbols is $1 / T_{1}$ measured for uniaxially aligned powder sample with NMR in $B_{\text {ext }}=9 \mathrm{~T}$ applied along the $a b$ plane for the superposed peak of ${ }^{63} \mathrm{Cu}\left(\mathrm{I}_{1}\right)$ and ${ }^{63} \mathrm{Cu}\left(\mathrm{I}_{2}\right)$ sites. (b) $1 / T_{1}^{\mathrm{Na}}$ observed for the interplanar ${ }^{23} \mathrm{Na}(\mathrm{I})$ sites in a powder sample of $\mathrm{Na}_{2} \mathrm{IrO}_{3}$ in $B_{\text {ext }}=5$ and $9 \mathrm{~T}$.

$\mathrm{Cu}\left(\mathrm{I}_{1,2}\right)$ sites due to their spatial proximity, and hence the magnitude of $1 / T_{1}$ at the ${ }^{63} \mathrm{Cu}(\mathrm{H})$ sites is larger by a factor of $\sim 3$. As noted above, this is the underlying reason why the ${ }^{63} \mathrm{Cu}(\mathrm{H}) \mathrm{NQR}$ signal detection becomes difficult above $\sim 70 \mathrm{~K}$. Otherwise, the temperature dependence of $1 / T_{1}$ is qualitatively the same between the ${ }^{63} \mathrm{Cu}(\mathrm{H}),{ }^{63} \mathrm{Cu}\left(\mathrm{I}_{1}\right)$, and ${ }^{63} \mathrm{Cu}\left(\mathrm{I}_{2}\right)$ sites.

$1 / T_{1}$ grows slightly below $300 \mathrm{~K}$, a typical signature of the gradual development of short-range spin-spin correlations, in the present case between Ir spins. It is followed by an onset of a dramatic suppression of $1 / T_{1}$ below $\sim 150 \mathrm{~K}$. A semilogarithmic plot in Fig. 7 (a) indicates that $1 / T_{1}$ follows an activation behavior over two decades, from $\sim 100 \mathrm{~K}$ to $\sim 20 \mathrm{~K}$, due to a complete suppression of the
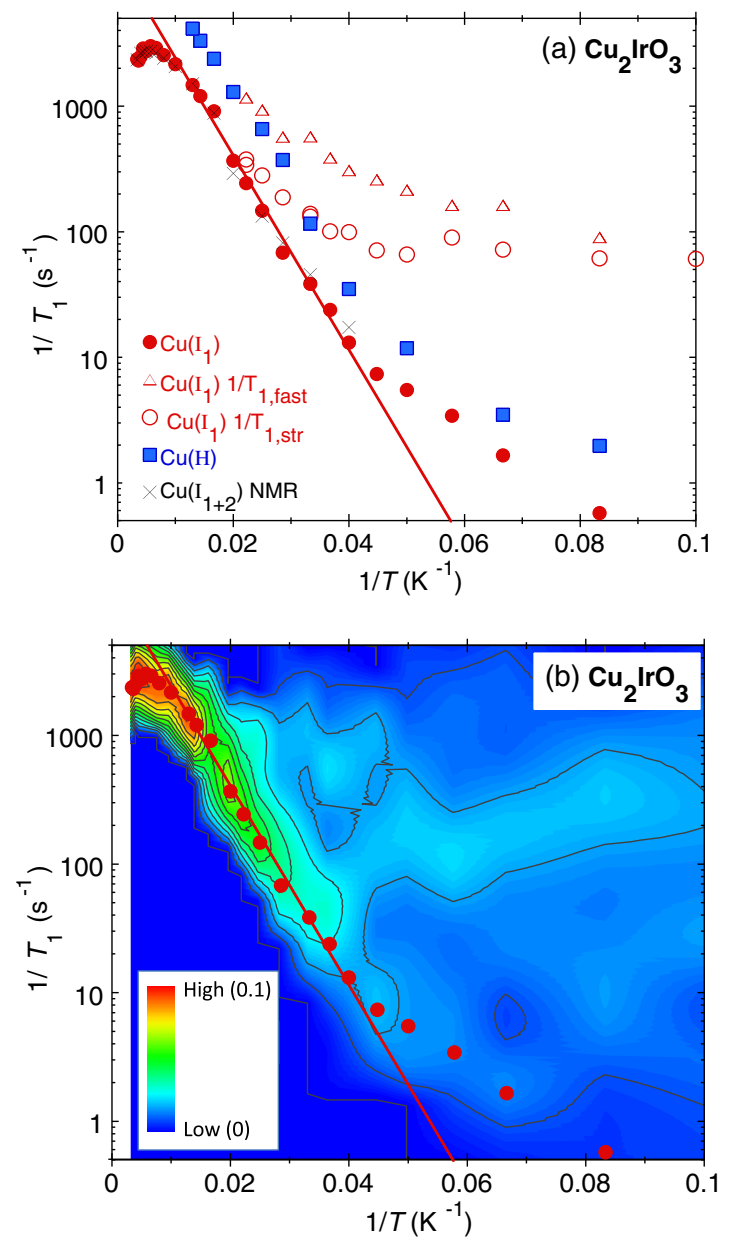

FIG. 7. (a) The same NQR and NMR $1 / T_{1}$ results as in Fig. 6(a), plotted as a function of inverse temperature $1 / T$ at the ${ }^{63} \mathrm{Cu}\left(\mathrm{I}_{1}\right)$ and ${ }^{63} \mathrm{Cu}(\mathrm{H})$ sites (filled symbols). The solid line shows an activation behavior, $1 / T_{1} \sim \exp \left(-\Delta / k_{B} T\right)$ with a gap, $\Delta / k_{B}=175 \pm 30 \mathrm{~K}$. Open triangles represent the fast component $1 / T_{1 \text {,fast }}$ in the two-component fit with Eq. (A3), while open bullets show the averaged behavior $1 / T_{1, \text { str }}$ as estimated from the stretched single exponential fit with Eq. (A2), both at the ${ }^{63} \mathrm{Cu}\left(\mathrm{I}_{1}\right)$ sites. (b) The color contour plot of the temperature dependence of the probability distribution $P\left(1 / T_{1}\right)$ of $1 / T_{1}$ at ${ }^{63} \mathrm{Cu}\left(\mathrm{I}_{1}\right)$ sites measured with $\mathrm{NQR}$, as determined by inverse Laplace transform of $M(t)$. We overlaid the NQR $1 / T_{1}$ at ${ }^{63} \mathrm{Cu}\left(\mathrm{I}_{1}\right)$ sites and its activation behavior, $1 / T_{1} \sim \exp \left(-\Delta / k_{B} T\right)$, from (a).

low-frequency component of the fluctuating hyperfine magnetic fields $h_{\text {perp }}$. By fitting the result to an activation form, $1 / T_{1} \sim \exp \left(-\Delta / k_{B} T\right)$, we estimate the gap, $\Delta / k_{B}=$ $175 \pm 30 \mathrm{~K}(\Delta \sim 15 \mathrm{meV})$.

We note that $1 / T_{1}$ develops a distribution below $\sim 60 \mathrm{~K}$, and hence the $1 / T_{1}$ results plotted below $\sim 60 \mathrm{~K}$ with filled symbols in Figs. 5(b), 6(a), and 7 represent the intrinsic slow component (i.e., $1 / T_{1, \text { slow }}$ ) estimated from the twocomponent fit with Eq. (A3), as explained in detail in Appendix A. We emphasize, however, that our key finding of the activation behavior in Fig. 7(a) does not depend on 
the details of how we estimate $1 / T_{1}$ from the nuclear spin recovery curve $M(t)$. The proof comes from the distribution function $P\left(1 / T_{1}\right)$ of $1 / T_{1}$ directly calculated from the inverse Laplace transform of the nuclear magnetization $M(t)[40,41]$, as explained in Sec. III C.

Also plotted in Figs. 5(b) and 7 using open bullets are $1 / T_{1, \text { str }}$ obtained from the phenomenological stretched fit of $M(t)$ with Eq. (A2) [42,43]. As explained in Sec. III C, $1 / T_{1, \text { str }}$ is a good measure of the center of gravity of the distributed $1 / T_{1}$. As the influence of inhomogeneous spin fluctuations from defects grows near $T_{f}$, the aforementioned activation behavior of the intrinsic slow component of $1 / T_{1}$ is terminated by the precursor of spin freezing, which also results in a shoulder of $1 / T_{1, \text { str }}$ below $\sim 30 \mathrm{~K}$, as shown in Fig. 5(b). This is followed by a gradual loss of ${ }^{63,65} \mathrm{Cu}$ NQR signal intensity below $T_{f} \sim 10 \mathrm{~K}$ in Fig. 5(a). The fact that half of the NQR signal remains observable without exhibiting any magnetic anomalies (such as the line broadening, divergence of $1 / T_{1}$, or signal loss) indicates that a majority of Ir spins themselves are not slowing down toward eventual long-range order or freezing. Instead, it is the inhomogeneously distributed defects (perhaps accompanied by minority Ir spins adjacent to them) that are freezing. This is consistent with the $\mu \mathrm{SR}$ observation that Ir spins remain dynamic even below $T_{f}$ [23,25], and also with a very wide distribution of $1 / T_{1}$ near $T_{f}$ as determined by inverse Laplace transform analysis in Sec. III C. Whether these defects originate from randomly distributed $\mathrm{Cu}^{2+}$ ions and/or clusters around the stacking faults encompassing several unit cells remains to be seen.

\section{Inverse Laplace transform of $M(t)$ for direct estimation of the $1 / T_{1}$ distribution}

A more precise, model-independent way to investigate the spin dynamics with $1 / T_{1}$ under the presence of distributions in the relaxation mechanisms is to take the inverse Laplace transform of $M(t)$ and directly deduce the distribution function $P\left(1 / T_{1}\right)$ of $1 / T_{1}[40,41]$ :

$$
M(t)=\sum_{i}\left[M_{o}-A \exp \left(-3 t / T_{1, i}\right)\right] P\left(1 / T_{1, i}\right),
$$

where we normalize the overall probability to 1 , $\sum_{i} P\left(1 / T_{1, i}\right)=1$. We numerically inverted $M(t)$ [41] utilizing Tikhonov regularization (i.e., a smoothing factor) $[44,45]$, and deduced the distribution function $P\left(1 / T_{1}\right)$ as summarized in Fig. 8 at representative temperatures.

We do not presume any phenomenological functional forms for $M(t)$, such as the existence of two separate components in Eq. (A3). Nonetheless, our $P\left(1 / T_{1}\right)$ results indicate that a new small side peak begins to emerge with fast values of $1 / T_{1}$ below $\sim 60 \mathrm{~K}$. The emergence of the two peak structure in $P\left(1 / T_{1}\right)$ indicates that the intrinsic, slow $T_{1}$ process begins to be short-circuited by the faster component(s) in the vicinity of defect spins as the intrinsic

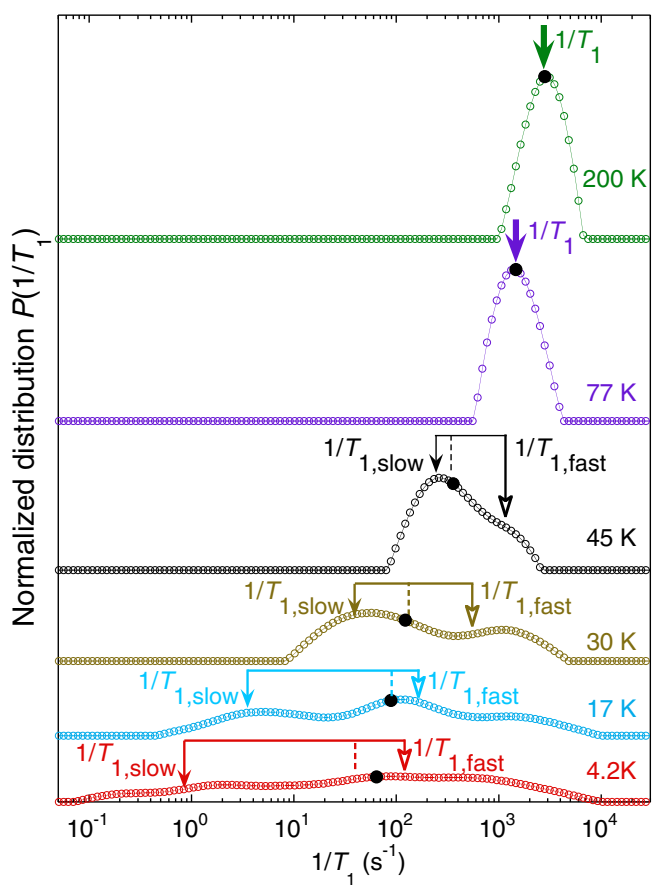

FIG. 8. Distribution function $P\left(1 / T_{1}\right)$ of $1 / T_{1}$ at representative temperatures directly computed from the recovery data $M(t)$ based on the inverse Laplace transform [41]. For clarity, the origin is shifted vertically except for $4.2 \mathrm{~K} . P\left(1 / T_{1}\right)$ is centered around a single value of $1 / T_{1}$ above $60 \mathrm{~K}$; the peak location at 77 and $200 \mathrm{~K}$ agrees very well with $1 / T_{1}$ estimated from the singlecomponent fit with Eq. (A1), as marked by thick arrows. This one peak distribution of $P\left(1 / T_{1}\right)$ breaks down at lower temperatures. The filled and open arrows at and below $45 \mathrm{~K}$ mark $1 / T_{1 \text {,slow }}$ and $1 / T_{1 \text {,fast }}$ obtained from the two-component fit with Eq. (A3), respectively. The dashed lines mark $1 / T_{1, \text { str }}$ estimated from Eq. (A2), which keeps track of the center of gravity of $P\left(1 / T_{1}\right)$ marked by black filled bullets.

$T_{1}$ slows down. Since the influence of the defect-induced fast relaxation depends on the distance with observed nuclear spins, the defect-induced fast component in $1 / T_{1}$ generally has a broad distribution.

To show the key features of $P\left(1 / T_{1}\right)$ visually, we plotted $P\left(1 / T_{1}\right)$ in a color contour map in Fig. $7($ b). We can identify the existence of the well-defined, slower component of $1 / T_{1}$ in the ridge structure (colored in red or green) that continues from $\sim 60 \mathrm{~K}$ down to $\sim 15 \mathrm{~K}$ (i.e., $1 / T=$ 0.015-0.07). On the other hand, the defect-induced fast contribution to $1 / T_{1}$ develops a wide distribution centered around $\sim 150 \mathrm{~s}^{-1}$, and remains roughly constant; see the light blue horizontal section starting from $1 / T \sim 0.04-0.1$ in Fig. 7(b). Interestingly, recent theoretical calculations of $1 / T_{1}$ for the Kitaev QSL predict that the presence of bond disorder leads to a constant $1 / T_{1}$ with a broad distribution [30]. We emphasize that our observation of the activation behavior for the intrinsic slow component and the roughly constant behavior induced by defects, both nicely captured in Fig. 7(b), does not depend on fitting procedures of $M(t)$, 
such as the stretched nature of the relaxation function in Eq. (A2) or the presence of two components in Eq. (A3).

Also notice that $1 / T_{1, \text { slow }}$ and $1 / T_{1 \text {,fast }}$ estimated from the two-component fit (marked by thin filled and thin open arrows, respectively, in Fig. 8) agree fairly well with the two peaks observed for $P\left(1 / T_{1}\right)$ in a wide temperature range except near and below $T_{f}$, where the two-component fit itself becomes invalid. This justifies the two-component fit with Eq. (A3) used above $T_{f}$ to generate intrinsic $1 / T_{1}$ as $1 / T_{1 \text {,slow }}$ in Figs. 5(b), 6(a), and 7. Interestingly, $1 / T_{1, \text { str }}$ estimated using Eq. (A2) (marked by vertical dashed lines in Fig. 8) agrees well with the center of gravity of the distribution $P\left(1 / T_{1}\right)$ (marked by black filled bullets). This means that $1 / T_{1 \text {,str }}$ probes only the spatially averaged behavior of the entire sample, and does not always represent the intrinsic behavior of $1 / T_{1}$.

\section{Comparison with low-energy Ir spin excitations of $\mathrm{Na}_{2} \mathrm{IrO}_{3}, \alpha-\mathrm{RuCl}_{3}$, and $\mathrm{H}_{3} \mathrm{LiIr}_{2} \mathrm{O}_{6}$}

It is illustrative to compare the $1 / T_{1}$ results of $\mathrm{Cu}_{2} \mathrm{IrO}_{3}$ with those observed for other Kitaev candidate materials. In Fig. 6(b), we summarize $1 / T_{1}^{\mathrm{Na}}$ measured for the interplanar ${ }^{23} \mathrm{Na}(\mathrm{I})$ sites of $\mathrm{Na}_{2} \mathrm{IrO}_{3}$ at the central peak of the powder ${ }^{23} \mathrm{Na}$ NMR line shape. We refer readers to Appendix $\mathrm{C}$ for the details of ${ }^{23} \mathrm{Na} \mathrm{NMR}$ measurements. Simutis et al. also reported analogous $1 / T_{1}^{\mathrm{Na}}$ data in ambient and applied pressure [46]. Their results, reported in a limited temperature range up to $25 \mathrm{~K}$, are in good agreement with ours. The overall magnitude of $1 / T_{1}^{\mathrm{Na}}$ is nearly 2 orders of magnitude slower than at the $\mathrm{Cu}\left(\mathrm{I}_{1,2}\right)$ sites in Fig. 6(a), because the hyperfine coupling $A_{\mathrm{hf}}$ is much smaller at the ${ }^{23} \mathrm{Na}$ sites, as evidenced by the ${ }^{23} \mathrm{Na}$ NMR Knight shift results [see Fig. 11(b) in Sec. III E].

Below $300 \mathrm{~K}$ down to $\sim 25 \mathrm{~K}, 1 / T_{1}^{\mathrm{Na}}$ gradually increases as the short-range $\mathrm{Ir}-\mathrm{Ir}$ spin correlations grow, without exhibiting a downturn observed for $\mathrm{Cu}_{2} \mathrm{IrO}_{3}$. The critical slowing-down of Ir spin fluctuations below $\sim 25 \mathrm{~K}$ toward the three-dimensional zigzag antiferromagnetic long-range order results in a sharp divergent behavior of $1 / T_{1}^{\mathrm{Na}}$ at $T_{N}=16.5 \pm 0.5 \mathrm{~K}$. This is consistent with the fact that the hyperfine form factor does not cancel at the ${ }^{23} \mathrm{Na}$ (I) sites for the zigzag order pattern [schematically shown in Fig. 2(b)]. Analogous divergent behavior is commonly observed for conventional antiferromagnets, such as $\mathrm{CuO}$ [47].

Below $T_{N}, 1 / T_{1}^{\mathrm{Na}}$ is quickly suppressed, accompanied by dramatic broadening of the ${ }^{23} \mathrm{Na}$ powder NMR line shape due to the static hyperfine magnetic fields from zigzag ordered Ir spins [see Fig. 17 in Appendix C, as well as Ref. [46] ]. In general, $1 / T_{1}$ in the antiferromagnetically ordered state below $T_{N}$ is dominated by a two- or threemagnon Raman process, resulting in suppression of $1 / T_{1}$ obeying a power law; it is generally followed by an activation behavior $1 / T_{1} \sim T^{2} \exp \left(-\Delta_{\text {magnon }} / k_{B} T\right)$ due to the anisotropy gap $\Delta_{\text {magnon }}$ for magnons at lower temperatures below $\Delta_{\text {magnon }} / k_{B} \quad[48,49]$. While our $1 / T_{1}^{\mathrm{Na}}$ results were measured for broad and superposed ${ }^{23} \mathrm{Na}$ NMR lines and do not allow us to conduct detailed analysis below $T_{N}$, the observed behavior of $1 / T_{1}^{\mathrm{Na}}$ below $T_{N}$ is similar to the conventional behavior expected for three-dimensional or quasi-two-dimensional antiferromagnets, such as $\mathrm{CuO}$ and $\mathrm{YBa}_{2} \mathrm{Cu}_{3} \mathrm{O}_{6}$ [49].

Our conventional findings on $\mathrm{Na}_{2} \mathrm{IrO}_{3}$ are no surprise, in view of the fact that earlier inelastic neutron scattering measurements established that the low-energy sector of the spin excitation spectrum in $\mathrm{Na}_{2} \mathrm{IrO}_{3}$ is dominated by magnons that are not native to the pure Kitaev Hamiltonian in Eq. (1) [7]. In this context, it is also interesting to compare our results with those previously reported for $\alpha-\mathrm{RuCl}_{3}$ [50-52], because magnons also dominate its spin excitation spectrum in the low-field regime [19-21]. Not surprisingly, $1 / T_{1}$ observed at the ${ }^{35} \mathrm{Cl}$ site of $\alpha-\mathrm{RuCl}_{3}$ in the low-field regime $B_{\text {ext }}=2.35 \mathrm{~T}$ with antiferromagnetic ground state [52] is similar to the conventional behavior observed here for $\mathrm{Na}_{2} \mathrm{IrO}_{3}$. Our NQR results for $\mathrm{Cu}_{2} \mathrm{IrO}_{3}$ in Figs. 6(a) and 7 are fundamentally different.

In the case of $\alpha-\mathrm{RuCl}_{3}$, application of a modest magnetic field above $\sim 7 \mathrm{~T}$ drives the antiferromagnetic ground state to a field-induced paramagnetic state with a gap [50-52]. This is often attributed to a spin liquid state. The report of the fractionalized thermal Hall effect [53] in the intermediate field regime of $\alpha-\mathrm{RuCl}_{3}$ as well as a peculiar magnetic field effect on magnon dispersion [21] certainly raises the hope that chiral spin liquid with Majorana edge modes may be induced by a magnetic field, but even the classical spin wave theory can semiquantitatively account for the former [54]. We also caution that application of an external magnetic field could fundamentally alter the nature of spin excitations of quantum spin systems. For example, $B_{\text {ext }}$ exceeding 5.3 $\mathrm{T}$ applied to the transverse field Ising chains realized in $\mathrm{CoNb}_{2} \mathrm{O}_{6}$ suppresses a magnetic long-range order and induces a spin excitation gap that manifests itself in the ${ }^{93} \mathrm{Nb}$ NMR properties [55]. The nature of the fieldinduced gap and its potential relation to Kitaev physics has been at the center of recent intense debate [21,50-53], and deserves further careful examination.

Finally, we compare $\mathrm{Cu}_{2} \mathrm{IrO}_{3}$ and $\mathrm{H}_{3} \mathrm{LiIr}_{2} \mathrm{O}_{6}$ [15]; both of these Kitaev candidate materials remain paramagnetic without undergoing magnetic long-range order. Interestingly, $1 / T_{1}$ observed at the intralayer ${ }^{7} \mathrm{Li}$ sites of $\mathrm{H}_{3} \mathrm{LiIr}_{2} \mathrm{O}_{6}$ shows qualitatively the same behavior as the intralayer ${ }^{63} \mathrm{Cu}(\mathrm{H})$ as well as interlayer ${ }^{63} \mathrm{Cu}\left(\mathrm{I}_{1}\right)$ sites from $300 \mathrm{~K}$ down to $\sim 50 \mathrm{~K}$, where the intrinsic behavior of ${ }^{7} \mathrm{Li} 1 / T_{1}$ appears to be taken over by large defect-induced contributions. On the other hand, $1 / T_{1}$ observed at the interlayer ${ }^{1} \mathrm{H}$ sites in $\mathrm{H}_{3} \mathrm{LiIr}_{2} \mathrm{O}_{6}$, which corresponds to the ${ }^{63} \mathrm{Cu}(\mathrm{I})$ sites in the present case, shows no hint of a gapped behavior at low temperatures. This apparent discrepancy from the ${ }^{7} \mathrm{Li}$ or our ${ }^{63} \mathrm{Cu}$ results, however, does not necessarily mean that $\mathrm{H}_{3} \mathrm{LiIr}_{2} \mathrm{O}_{6}$ does 
not have analogous activation behavior of $1 / T_{1}$. It is well known that ${ }^{1} \mathrm{H}$ NMR $1 / T_{1}$ results in herbertsmithite kagome antiferromagnet $\mathrm{ZnCu}_{3}(\mathrm{OH})_{6} \mathrm{Cl}_{2}$ [32] are dominated by defect spins, especially in low magnetic fields, and do not resemble the intrinsic $1 / T_{1}$ results observed at ${ }^{63} \mathrm{Cu}$ [32] or ${ }^{17} \mathrm{O}$ [34] sites. This is simply because Fermi's contact hyperfine coupling of ${ }^{1} \mathrm{H}$ nuclear spin through the $1 s$ orbital is generally very weak, and hence $1 / T_{1}$ measured at ${ }^{1} \mathrm{H}$ sites is comparatively more sensitive to defect-induced spin fluctuations. We note that the ${ }^{1} \mathrm{H}$ as well as ${ }^{7} \mathrm{Li}$ NMR linewidth in $\mathrm{H}_{3} \mathrm{LiIr}_{2} \mathrm{O}_{6}$ is greater than the small NMR frequency shift (i.e., the Knight shift is very small). This suggests that a large fraction of nuclear spins have stronger hyperfine couplings with defect spins than with nearby $\operatorname{Ir}^{4+}$ spins with intrinsic Kitaev properties, which explains why ${ }^{7} \mathrm{Li}$ and ${ }^{1} \mathrm{H}$ NMR $1 / T_{1}$ results are overwhelmed by defect contributions. In the case of $\mathrm{Cu}_{2} \mathrm{IrO}_{3}$, this does not happen, because the transferred hyperfine couplings of ${ }^{63} \mathrm{Cu}$ nuclear spins through the $4 s$ orbital [31] with surrounding Ir sites are larger by an order of magnitude, as demonstrated in the next section.

\section{E. NMR measurements in applied magnetic field}

We also conducted high-field NMR measurements using a uniaxially aligned powder sample of $\mathrm{Cu}_{2} \mathrm{IrO}_{3}$ cured in a glue (Stycast 1266) in the presence of $B_{\text {ext }}=9$ T. This is a standard trick used in powder NMR experiments, and creates a pseudo-single-crystal by taking advantage of the anisotropy of magnetization in high magnetic fields [38]. We summarize representative ${ }^{63} \mathrm{Cu}$ NMR line shapes observed for the nuclear spin $I_{z}=+1 / 2$ to $-1 / 2$ central transition of the aligned powder sample in $B_{\text {ext }}=9 \mathrm{~T}$ applied along the aligned $c$ and $a b$ axis in Figs. 9(b) and 9(c), respectively. See Fig. 15 in Appendix B for the field sweep NMR spectra covering the satellite transitions between the nuclear spin $I_{z}= \pm 1 / 2$ to $\pm 3 / 2$ states. From the comparison with the NMR line shapes for unaligned powder in Fig. 9(a), we estimate the fraction of the uniaxially aligned powder as approximately $50 \%$; the rest of the powder remains randomly oriented.

We could assign the ${ }^{63,65} \mathrm{Cu}$ NMR peaks and shoulders arising from $\mathrm{Cu}\left(\mathrm{I}_{1,2}\right)$ sites in aligned and unaligned portions of the sample, as marked by arrows and + and $\times$ symbols in Figs. 9(a)-9(c). However, identification of the NMR signals from less abundant ${ }^{63} \mathrm{Cu}(\mathrm{H})$ sites proved to be a major challenge, because the ${ }^{63,65} \mathrm{Cu}(\mathrm{H})$ central transition is shifted by the second- and higher-order effects caused by the extremely large nuclear quadrupole frequency $\left({ }^{63} \nu_{\mathrm{NQR}} \sim 52 \mathrm{MHz}\right)$, and superposed by much stronger central and satellite transitions of the ${ }^{63,65} \mathrm{Cu}\left(\mathrm{I}_{1,2}\right)$ sites. Moreover, since ${ }^{63} \nu_{\mathrm{NQR}}$ at the ${ }^{63} \mathrm{Cu}(\mathrm{H})$ sites happens to be almost exactly twice larger than ${ }^{63} \nu_{\mathrm{NQR}}$ at the ${ }^{63} \mathrm{Cu}\left(\mathrm{I}_{1,2}\right)$ sites, the small $I_{z}= \pm 3 / 2$ to $\pm 1 / 2$ satellite peaks of the ${ }^{63} \mathrm{Cu}(\mathrm{H})$ sites are accidentally superposed by the satellite
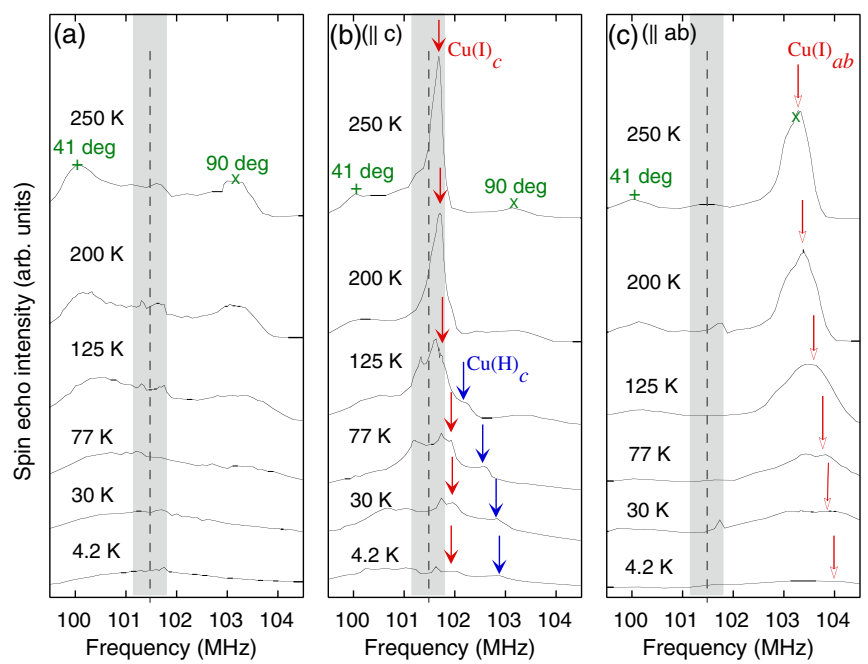

FIG. 9. (a) Representative ${ }^{63} \mathrm{Cu}$ NMR line shapes observed for an unaligned powder sample in an external magnetic field $B_{\text {ext }}=9 \mathrm{~T}$. Green $\times$ and + mark the peaks corresponding to the particles in which $B_{\text {ext }}$ is 90 -deg (i.e., $a b$ plane) and 41-deg tilted from the main principal axis of the electric field gradient tensor along the $c$ axis. The gray shaded region is superposed by weak ${ }^{23} \mathrm{Na}$ NMR signals centered at $\sim 101.3 \mathrm{MHz}$ arising from a small amount of impurity phase and the background ${ }^{63} \mathrm{Cu}$ NMR signals at $\sim 101.7 \mathrm{MHz}$ from the metallic parts used in our cryogenic NMR probe. Dashed vertical line shows the unshifted ${ }^{63} \mathrm{Cu}$ NMR frequency ${ }^{63} \nu_{o}=\gamma_{n} B_{\text {ext }}$. (b) Representative ${ }^{63} \mathrm{Cu}$ NMR line shapes observed for a uniaxially aligned powder sample in $B_{\text {ext }}=9 \mathrm{~T}$ applied along the aligned $c$ axis. Red filled arrows mark the ${ }^{63} \mathrm{Cu}(\mathrm{I})$ peak, while the blue filled arrows mark the ${ }^{63} \mathrm{Cu}(\mathrm{H})$ peak, both in the $B_{\text {ext }} \| c$ geometry. (c) Representative ${ }^{63} \mathrm{Cu}$ NMR line shapes observed for a uniaxially aligned powder sample in $B_{\text {ext }}=9 \mathrm{~T}$ applied along the aligned $a b$ plane. Red open arrows mark the ${ }^{63} \mathrm{Cu}(\mathrm{I})$ peak in $B_{\text {ext }} \| a b$ geometry.

structures arising from the ${ }^{63} \mathrm{Cu}\left(\mathrm{I}_{1,2}\right)$ sites. To make matters worse, defect spins polarized by $B_{\text {ext }}$ broaden all the NMR lines below $\sim 50 \mathrm{~K}$, where the ${ }^{63} \mathrm{Cu}(\mathrm{H}) \mathrm{NQR}$ signal was readily observable. Nonetheless, we were able to resolve the ${ }^{63} \mathrm{Cu}(\mathrm{H})$ NMR central transition peak below $\sim 120 \mathrm{~K}$ in the $B_{\text {ext }} \| c$ geometry, as marked by blue arrows in Fig. 9(b).

The resonance frequency of the ${ }^{63} \mathrm{Cu}\left(\mathrm{I}_{1,2}\right)$ and ${ }^{63} \mathrm{Cu}(\mathrm{H})$ peak for $B_{\text {ext }} \| c$ in Fig. 9(b) is shifted from the bare Zeeman frequency ${ }^{63} \nu_{o}$ marked by the vertical dashed line due to the paramagnetic NMR Knight shift, ${ }^{63} \mathrm{~K}=$ $N_{\text {nn }} A_{\text {hf }} \chi_{\text {spin }} / N_{A} \mu_{B}$, where $N_{\text {nn }}$ is the number of the nearestneighbor Ir sites that induce transferred hyperfine magnetic fields at $\mathrm{Cu}$ nuclear spins, $N_{A}$ is Avogadro's number, $\mu_{B}$ is the Bohr magneton, $\chi_{\text {spin }}$ is the spin contribution to the bulk susceptibility, $\chi=\chi_{\text {spin }}+\chi_{\text {dia }}+\chi_{v v}$, where the diamagnetic term $\chi_{\mathrm{dia}} \sim-0.09 \times 10^{-3} \mathrm{emu} / \mathrm{mol}$ from the standard table, and $\chi_{v v} \sim 0.16 \times 10^{-3} \mathrm{emu} / \mathrm{mol}$ [18]. ${ }^{63} \mathrm{Cu}\left(\mathrm{I}_{1,2}\right)$ sites are bonded with two $\mathrm{O}$ sites above and below [see Fig. 1(e)], each of which bonds with two Ir sites, 
resulting in $N_{\mathrm{nn}}=4$. On the other hand, $N_{\mathrm{nn}}=6$ for ${ }^{63} \mathrm{Cu}(\mathrm{H})$, as may be seen in Fig. $1(\mathrm{~d})$.

In the case of the ${ }^{63} \mathrm{Cu}\left(\mathrm{I}_{1,2}\right)$ peak in $B_{\text {ext }} \| a b$, the apparent Knight shift ${ }^{63} K_{\text {apparent }}$ is also affected by the second-order contribution of the nuclear quadrupole interaction $\Delta \nu_{Q}^{(2)}$, which is proportional to $1 /\left(\gamma_{n} B_{\text {ext }}\right)^{2}$. We therefore measured ${ }^{63} K_{\text {apparent }}$ at different magnetic field values, linearly extrapolated the results to the high-field limit of $1 /\left(\gamma_{n} B_{\text {ext }}\right)^{2}=0$ [38], and estimated ${ }^{63} \mathrm{~K}$ as shown in Fig. 10. On the other hand, within experimental uncertainties, we found no dependence of ${ }^{63} K_{\text {apparent }}$ on $1 /\left(\gamma_{n} B_{\text {ext }}\right)^{2}$ for both ${ }^{63} \mathrm{Cu}\left(\mathrm{I}_{1,2}\right)$ and ${ }^{63} \mathrm{Cu}(\mathrm{H})$ sites in the $B_{\text {ext }} \| c$ geometry. This implies that the nuclear quadrupole effects $\Delta \nu_{Q}^{(2)}$ play no significant roles in the NMR frequency shift for $B_{\text {ext }} \| c$, because the main principal axis of the EFG tensor is along the $c$ axis and the asymmetry parameter $\eta$ of the EFG tensor is negligible $(\eta \sim 0)$. This is consistent with the local symmetry of these sites.

We summarize the temperature dependence of ${ }^{63} \mathrm{~K}$ in Fig. 11(a) in comparison to $\chi_{\text {spin }}$ measured in $5 \mathrm{~T}\left(\chi_{\text {spin }}\right.$ does not depend on magnetic field above $\sim 30 \mathrm{~K}[22])$. ${ }^{63} \mathrm{~K}$ increases with decreasing temperature, and saturates below $\sim 30 \mathrm{~K}$. Interestingly, ${ }^{63} \mathrm{~K}$ observed for $\mathrm{Cu}_{2} \mathrm{IrO}_{3}$ shows qualitatively the same site and temperature dependencies as the ${ }^{23} \mathrm{Na}$ NMR shift ${ }^{23} \mathrm{~K}$ observed for $\mathrm{Na}_{2} \mathrm{IrO}_{3}$ in the paramagnetic state above $T_{N} \sim 16.5 \mathrm{~K}$, as shown in Fig. 11(b). Noting that ${ }^{63} \mathrm{~K}$ probes local spin susceptibility rather than the bulk averaged result, our ${ }^{63} \mathrm{~K}$ results indicate that the upturn of the bulk $\chi$ data observed below $\sim 30 \mathrm{~K}$ does not reflect the intrinsic behavior of the Ir honeycomb planes, but should be attributed to defect spins. It is the enhanced local spin susceptibility of the defect spins that

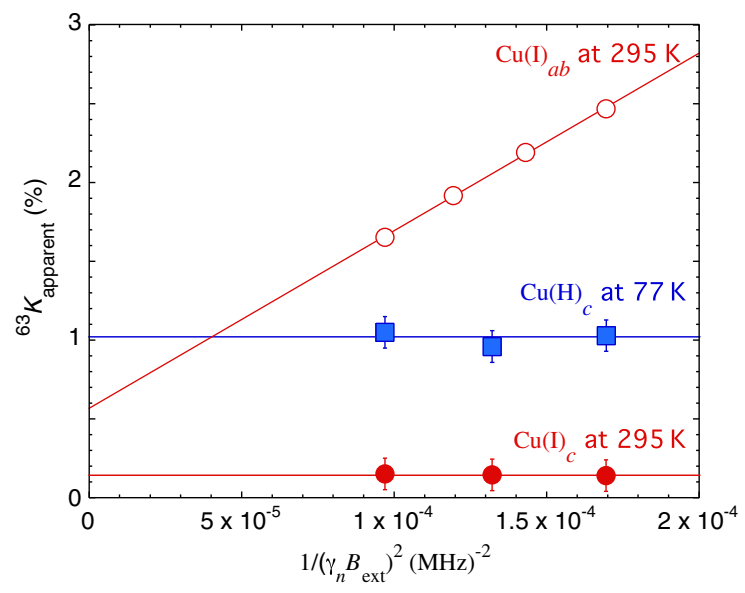

FIG. 10. Separation of the NMR Knight shift ${ }^{63} \mathrm{~K}$ contribution from the second-order quadrupole contribution $\Delta \nu_{Q}^{(2)}$ based on the measurements of ${ }^{63} K_{\text {apparent }}$ in several magnetic fields. The linear extrapolation of ${ }^{63} K_{\text {apparent }}$ to the limit of $1 /\left(\gamma_{n} B_{\text {ext }}\right)^{2}=0$ gives ${ }^{63} \mathrm{~K}$.
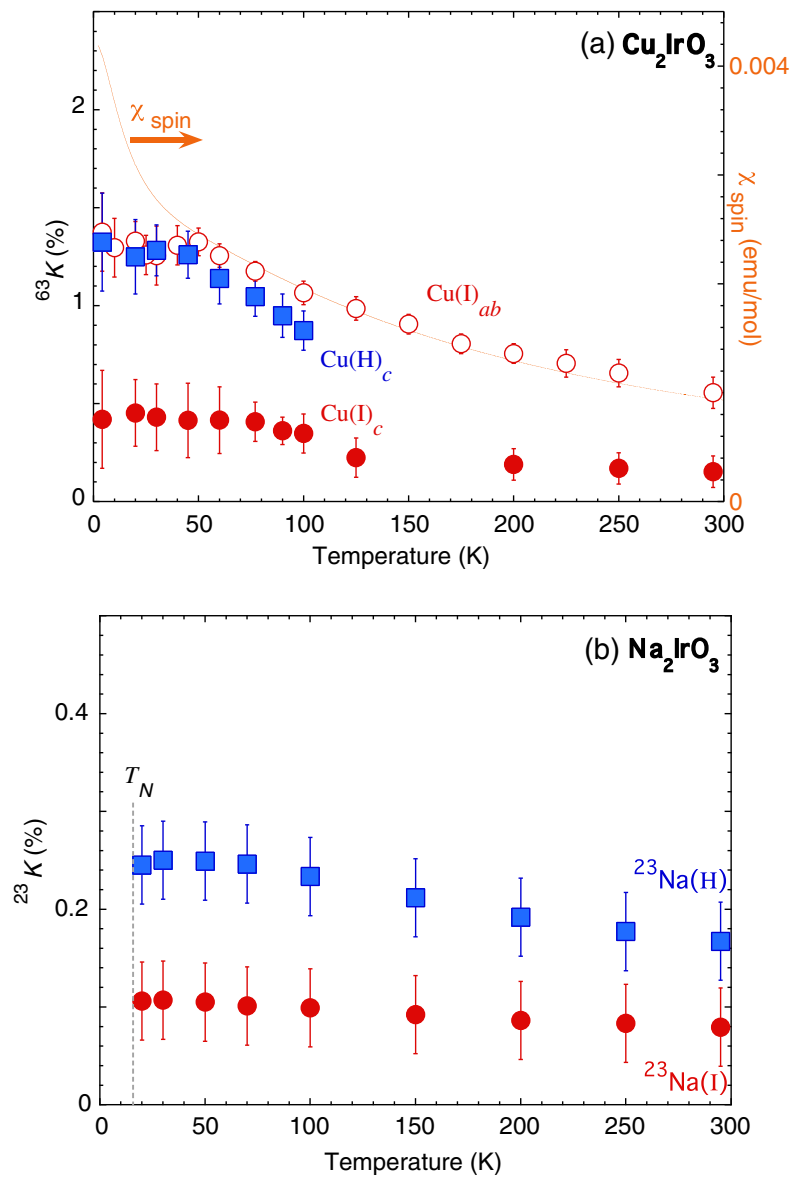

FIG. 11. (a) The temperature dependence of the intrinsic local spin susceptibility deduced as ${ }^{63} \mathrm{~K}$, in comparison to the bulk averaged susceptibility $\chi_{\text {spin }}$ measured in $5 \mathrm{~T}$ (right axis). Notice that the large upturn observed below $\sim 30 \mathrm{~K}$ for the bulk $\chi$ spin data is absent in ${ }^{63} \mathrm{~K}$. (b) The temperature dependence of the intrinsic local spin susceptibility deduced as ${ }^{23} \mathrm{~K}$ in $\mathrm{Na}_{2} \mathrm{IrO}_{3}$, as determined from the NMR line shapes measured for a powder sample in Fig. 17.

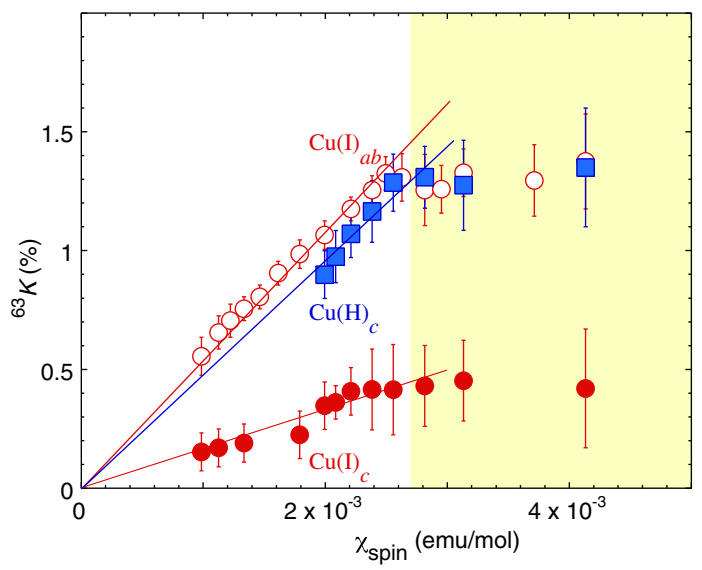

FIG. 12. The plot of ${ }^{63} \mathrm{~K}$ versus the spin contribution $\chi_{\text {spin }}$ for $\mathrm{Cu}_{2} \mathrm{IrO}_{3}$, choosing temperature as the implicit parameter. The solid lines represent the best liner fit through the origin. $\chi_{\text {spin }}$ is enhanced by defect spin contributions in the yellow shaded region, where the linearity breaks down. 
inhomogeneously broadens our high-field NMR peaks at low temperatures.

We plotted ${ }^{63} \mathrm{~K}$ versus $\chi_{\text {spin }}$ choosing temperature as the implicit parameter in Fig. 12, and estimated the hyperfine coupling from the slope of the linear fit as $A_{\mathrm{hf}}=$ $\left(N_{A} \mu_{B} / N_{\mathrm{nn}}\right) d\left({ }^{63} \mathrm{~K}\right) / d \chi_{\text {spin }}=2.3$ and $7.6 \mathrm{kOe} / \mu_{B}$ for ${ }^{63} \mathrm{Cu}\left(\mathrm{I}_{1,2}\right)$ in the $B_{\text {ext }} \| c$ and $\| a b$ geometry, respectively, and $A_{\mathrm{hf}}=4.4 \mathrm{kOe} / \mu_{B}$ for ${ }^{63} \mathrm{Cu}(\mathrm{H})$ in $B_{\text {ext }} \| c$. We were unable to determine $A_{\mathrm{hf}}$ at the ${ }^{63} \mathrm{Cu}(\mathrm{H})$ sites along the $a b$ direction, because the small central peak is buried underneath the ${ }^{65} \mathrm{Cu}\left(\mathrm{I}_{1,2}\right)$ signals due to extremely large $\Delta \nu_{Q}^{(2)}$, as explained earlier.

\section{DISCUSSIONS}

The activation behavior of $1 / T_{1}$ is generically observed when the spin excitation spectrum represented by $S(\mathbf{q}, E)$ has a gap in the low-energy sector. For example, the collective spin-singlet state realized in the two-leg spin ladders $\mathrm{SrCu}_{2} \mathrm{O}_{3}$ [56] and $\mathrm{La}_{6} \mathrm{Ca}_{8} \mathrm{Cu}_{24} \mathrm{O}_{41}$ [57] has a spin excitation gap as large as $\Delta / k_{B} \sim 450 \mathrm{~K}\left(\sim J_{H} / 2\right.$ along the rung formed by a pair of $\mathrm{Cu}$ ions), and exhibits an activation behavior of $1 / T_{1}$ at both ${ }^{63} \mathrm{Cu}[56,57]$ and ${ }^{17} \mathrm{O}$ [57] sites below $\sim 500 \mathrm{~K}$. Our $1 / T_{1}$ results in Fig. 7 are therefore consistent with the presence of a gap $\Delta / k_{B}=$ $175 \pm 30 \mathrm{~K}(\Delta \sim 15 \mathrm{meV})$ in the primary mode of the intrinsic spin excitation spectrum in $\mathrm{Cu}_{2} \mathrm{IrO}_{3}$. Note that this gap is comparable with the Ising interaction energy scale estimated above, $\left|J_{K}\right|=17-30 \mathrm{meV}$. In the pure Kitaev model, earlier theoretical analyses showed that the dispersion of the spin-1/2 excitations of fractionalized Majorana fermions may indeed have a gap as large as $\Delta_{\text {Majorana }} \sim 1.2 J_{K}[8,10]$. It is therefore tempting to associate our finding with the gapped excitations expected for Majorana fermions.

Broad high-energy spin excitations centered around $\mathbf{q}=\mathbf{0}$ with a gap $\Delta \sim 1.2\left|J_{K}\right|$ were previously reported for $\alpha-\mathrm{RuCl}_{3}$ by inelastic neutron scattering, but the low-energy sector is filled by additional spin-wave-like excitations [19,20]. In the present case, our observation of the faster component in $1 / T_{1}$ in the recovery curve $M(t)$ indicates the presence of an additional, spatially inhomogeneous relaxation mechanism(s). Theoretically, excitations of a pair of gauge fluxes with total spin 1 could short-circuit the slower NMR relaxation process in the pure Kitaev model, and such gauge flux excitations might morph into the spin waves (also with net spin 1) under the perturbation caused by the Heisenberg exchange term. It is not clear what roles the gauge fluxes and/or spin waves may be playing in our results. On the other hand, the growing distribution of $P\left(1 / T_{1}\right)$ toward $T_{f}$ seems to suggest that the faster component of $1 / T_{1}$ originates primarily from extrinsic defect spins.

It is important to distinguish our observation of the gapped behavior in zero magnetic field from earlier observations of a small field-induced gap in $\alpha-\mathrm{RuCl}_{3}$ [50-52]. To test the potential influence of the applied magnetic field on the gapped behavior observed for $\mathrm{Cu}_{2} \mathrm{IrO}_{3}$, we measured $1 / T_{1}$ at the lower satellite transition of the ${ }^{63} \mathrm{Cu}\left(\mathrm{I}_{1,2}\right)$ sites in $B_{\text {ext }}=9 \mathrm{~T}$ applied along the aligned $a b$ plane. In this field geometry, $1 / T_{1}$ probes the fluctuations of $h_{\text {perp }}$ along both the $a b$ plane and the $c$ axis. We summarize the NMR results with $\times$ symbols in Figs. 6(a) and 7(a). Comparison with the NQR results indicates that the gapped behavior of $1 / T_{1}$ does not depend significantly on the magnetic field, at least up to $9 \mathrm{~T}$.

A potential caveat in our interpretation of the gapped behavior of $S(\mathbf{q}, E)$ is that, in principle, $1 / T_{1}$ can be suppressed at low temperatures if the form factor satisfies $A_{\mathrm{hf}}\left(\mathbf{q}_{\mathrm{AFM}}\right)=0$ at a staggered vector $\mathbf{q}_{\mathrm{AFM}}$, where $S\left(\mathbf{q}_{\mathrm{AFM}}, E_{n}\right)$ grows due to short-range spin-spin correlations [39]. In this alternate scenario, Ir spins on the entire honeycomb planes continuously grow strong short-range order down to $T_{f}$ without developing long-range order, resulting in diminishing $1 / T_{1}$ due to geometrical cancellation of $A_{\mathrm{hf}}\left(\mathbf{q}_{\mathrm{AFM}}\right)$ by the high symmetry at $\mathrm{Cu}$ sites.

To illustrate the underlying mechanism in this alternate scenario, we present the possible short-range order patterns of Ir spins [7] in Figs. 1(b) and 1(c). Unlike $\mathrm{Na}_{2} \mathrm{IrO}_{3}$ with strong zigzag short-range order, $1 / T_{1}$ in $\mathrm{Cu}_{2} \mathrm{IrO}_{3}$ does not grow below $\sim 150 \mathrm{~K}$. Accordingly, we can rule out strong zigzag short-range order for $\mathrm{Cu}_{2} \mathrm{IrO}_{3}$. However, if Ir spins develop extremely strong Néel-type short-range spin-spin correlations shown in Fig. 1(c), the transferred hyperfine field $h_{\text {perp }}$ from six nearest-neighbor Ir sites of ${ }^{63} \mathrm{Cu}(\mathrm{H})$ would cancel out, because the sign of $h_{\text {perp }}$ alternates between six nearest neighbors. Although the location of the ${ }^{63} \mathrm{Cu}\left(\mathrm{I}_{1,2}\right)$ sites is somewhat shifted from the midpoint between two adjacent Ir sites, $h_{\text {perp }}$ should also nearly cancel out for ${ }^{63} \mathrm{Cu}\left(\mathrm{I}_{1,2}\right)$ sites. Thus, in principle, Néel-type short-range spin-spin correlations can suppress $1 / T_{1}$ in $\mathrm{Cu}_{2} \mathrm{IrO}_{3}$. However, we recall that such perfect cancellation of $h_{\text {perp }}$ expected for $1 / T_{1} \sim 0$ observed here generally requires extremely long spin-spin correlation length $\xi$. For example, in the case of ${ }^{17} \mathrm{O}$ NMR measurements in the square-lattice Heisenberg antiferromagnet $\mathrm{Sr}_{2} \mathrm{CuO}_{2} \mathrm{Cl}_{2}$ with no spin excitation gap above $T_{N}$ [58], $\xi$ reaches tens of lattice spacings [59] when $1 / T_{1}$ is completely suppressed due to the high symmetry at the ${ }^{17} \mathrm{O}$ sites. In view of the frustrated geometry of Ir spins in the present case, validity of such a scenario starting from as high as $\sim 150 \mathrm{~K}$ (comparable to $\sim\left|J_{K}\right| / k_{B}$, and much higher than $\left|J_{H}\right| / k_{B}$ ) seems somewhat remote. In addition, the intrinsic uniform spin susceptibility deduced from ${ }^{63} \mathrm{~K}$ in Fig. 11(a) does not exhibit any hint of a downturn below $\sim 150 \mathrm{~K}$, which is expected for such strong quasi-two-dimensional short-range Néel order. It is also worth recalling that ${ }^{17} \mathrm{O}$ as well as ${ }^{63} \mathrm{Cu}$ NMR $1 / T_{1}$ measurements of two-leg spin ladders in $\mathrm{La}_{6} \mathrm{Ca}_{8} \mathrm{Cu}_{24} \mathrm{O}_{41}$ successfully singled out the gapped spin 
excitation mode at $\sim 40 \mathrm{meV}$, even though $A_{\mathrm{hf}}(\mathbf{q})$ cancels at the ${ }^{17} \mathrm{O}$ sites [57].

Next, we wish to address the nature of defect spins and their influence on the NMR properties. As noted in Sec. I, recent XANES measurements for $\mathrm{Cu}_{2} \mathrm{IrO}_{3}$ showed that up to $\sim 1 / 3$ of ${ }^{63} \mathrm{Cu}(\mathrm{H})$ sites are occupied by $\mathrm{Cu}^{2+}$ ions rather than $\mathrm{Cu}^{+}$ions. On the other hand, the linear fit of the ${ }^{63} \mathrm{~K}$ versus $\chi$ plot in Fig. 12 extrapolates to the origin for both ${ }^{63} \mathrm{Cu}(\mathrm{H})$ and ${ }^{63} \mathrm{Cu}\left(\mathrm{I}_{1,2}\right)$ sites. This means that the van Vleck contribution to the Knight shift is vanishingly small, as expected for $\mathrm{Cu}^{+}$ions with filled $3 d$ orbitals. If the observed ${ }^{63} \mathrm{Cu}(\mathrm{H})$ NMR signals arise from $\mathrm{Cu}^{2+}$ ions, the intercept with the vertical axis of the extrapolated linear fit would be as large as $0.3 \%-1.5 \%$ due to the temperatureindependent van Vleck contribution for $\mathrm{Cu}^{2+}$ ions [31].

The lack of $\mathrm{Cu}^{2+}$ contributions in our ${ }^{63} \mathrm{Cu}(\mathrm{H})$ NMR data may be easily understood, if we recall that NMR signals at paramagnetic cation sites are generally not observable due to extremely fast NMR relaxation rates. In fact, ${ }^{63} \mathrm{Cu} \mathrm{NQR}$ and NMR signals in the paramagnetic state of the aforementioned $\mathrm{ZnCu}_{3}(\mathrm{OH})_{6} \mathrm{Cl}_{2}$ [32], $\mathrm{CuO}$ [47], $\mathrm{SrCu}_{2} \mathrm{O}_{3}$ [56], $\mathrm{La}_{6} \mathrm{Ca}_{8} \mathrm{Cu}_{24} \mathrm{O}_{41}$ [57], $\mathrm{Sr}_{2} \mathrm{CuO}_{2} \mathrm{Cl}_{2}$ [58], etc., are observable only because exchange narrowing effects induced by strong antiferromagnetic exchange interaction $J_{H} / k_{B}$ of the order of $200-1700 \mathrm{~K}$ between cation sites suppresses the NMR relaxation rates, which certainly would not be the case for the $\mathrm{Cu}^{2+}$ defect spins in $\mathrm{Cu}_{2} \mathrm{IrO}_{3}$. We therefore conclude that the primary contributions of $\mathrm{Cu}^{2+}$ defect spins to our NMR results are through the line broadening effects for the observable ${ }^{63} \mathrm{Cu}(\mathrm{H})$ and ${ }^{63} \mathrm{Cu}\left(\mathrm{I}_{1,2}\right)$ NMR signals below $\sim 50 \mathrm{~K}$ and the enhancement of $1 / T_{1}$ in their vicinity. The latter leads to a large distribution of $1 / T_{1}$, as seen in $P\left(1 / T_{1}\right)$ in Figs. 7(b) and 8.

\section{SUMMARY AND CONCLUSIONS}

We have reported comprehensive ${ }^{63} \mathrm{Cu}$ NQR and NMR measurements in $\mathrm{Cu}_{2} \mathrm{IrO}_{3}$, a proximate Kitaev QSL material that does not undergo magnetic long-range order. We showed that the intrinsic uniform spin susceptibility $\chi_{\text {spin }}$ of $\mathrm{Cu}_{2} \mathrm{IrO}_{3}$ shows nearly identical behavior as in the paramagnetic state of antiferromagnetic $\mathrm{Na}_{2} \mathrm{IrO}_{3}$ above $T_{N} \sim 17 \mathrm{~K}$. On the other hand, the low-energy sector of Ir spin excitations reflected on the temperature dependence of $1 / T_{1}$ is very different in $\mathrm{Cu}_{2} \mathrm{IrO}_{3} .1 / T_{1}$ shows activation behavior and is qualitatively different from the conventional behavior exhibited by antiferromagnetic $\mathrm{Na}_{2} \mathrm{IrO}_{3}$. Our finding strongly suggests that the low-energy sector of the spin excitation spectrum in $\mathrm{Cu}_{2} \mathrm{IrO}_{3}$ is dominated by a mode with a gap comparable to the magnitude of Ising interaction $\left|J_{K}\right|$, which may be related to gapped excitations expected for fractionalized Majonara fermions perturbed by the Heisenberg exchange term. In such an idealized scenario of the Kitaev lattice, it is not clear what roles the excitations of a pair of gauge fluxes might play. An analogous dilemma was previously encountered in the analysis of the spin excitations of $\alpha-\mathrm{RuCl}_{3}$, because the low-energy sector is dominated by magnons rather than gauge fluxes [20]. From the measurements of $1 / T_{1}$ alone, we cannot entirely rule out an alternate scenario that Ir spinspin correlation develops strong Néel-type short-range order with divergently long spin-spin correlation length. However, that would normally require suppression of $\chi_{\text {spin }}$ in quasi-two-dimensional Heisenberg antiferromagnets, and we did not find such a signature in our ${ }^{63} \mathrm{~K}$ results.

\section{ACKNOWLEDGMENTS}

The authors thank S.-S. Lee, R. Moessner, M. J. Graf, I. Kimchi, S. M. Winter, N. Perkins, Y. Motome, Y. B. Kim, and C. Varma for helpful discussions and communications. T. I. is supported by NSERC. F. T. is supported by NSF under DMR-1708929. P. M. S. is supported by The Rice University Consortium for Processes in Porous Media.

\section{APPENDIX A: FITTING PROCEDURES FOR 1/ $\boldsymbol{T}_{1}$}

The results of the nuclear magnetization $M(t)$ for the $1 / T_{1}$ measurements at ${ }^{63} \mathrm{Cu}\left(\mathrm{I}_{1}\right),{ }^{63} \mathrm{Cu}\left(\mathrm{I}_{2}\right)$, and ${ }^{63} \mathrm{Cu}(\mathrm{H})$ sites are qualitatively similar, and we applied the same fitting procedures. In what follows, we focus on the results for the ${ }^{63} \mathrm{Cu}\left(\mathrm{I}_{1}\right)$ sites that have the highest signal-to-noise ratio. In Figs. 13(a) and 13(b), we summarize $T_{1}$ recovery curves $M(t)$ observed for the ${ }^{63} \mathrm{Cu}\left(\mathrm{I}_{1}\right)$ site at selected temperatures. For the ${ }^{63} \mathrm{Cu}$ NQR measurements between the $I_{z}= \pm 3 / 2$ and $\pm 1 / 2$ states in zero magnetic field, magnetic transition would result in the exponential form of the recovery function [36,37],

$$
M(t)=M_{o}-A \exp \left(-3 t / T_{1}\right),
$$

where $1 / T_{1}, M_{o}$, and $A$ are the fitting parameters. We found that this single exponential form fits the recovery data nearly perfectly above $\sim 60 \mathrm{~K}$, as shown by dashed straight lines.

Below $\sim 60 \mathrm{~K}, 1 / T_{1}$ begins to exhibit a distribution and hence the fit with Eq. (A1) becomes progressively poorer. As a remedy, one can introduce a phenomenological stretched exponent $\beta$ introduced for randomly diluted antiferromagnets $[42,43]$ :

$$
M(t)=M_{o}-A \exp \left(-\left[3 t / T_{1, \mathrm{str}}\right]^{\beta}\right) .
$$

If there is no distribution in the relaxation process, the stretched fit would give $1 / T_{1, \text { str }}=1 / T_{1}$ and $\beta=1$. As summarized in Fig. 13(c), the stretched exponent begins to deviate from $\beta=1$ below $\sim 60 \mathrm{~K}$ as the intrinsic relaxation process slows down and becomes susceptible to the faster defect contributions. $1 / T_{1, \text { str }}$ results are approximate, but tend to elucidate the sample averaged behavior, as proven 

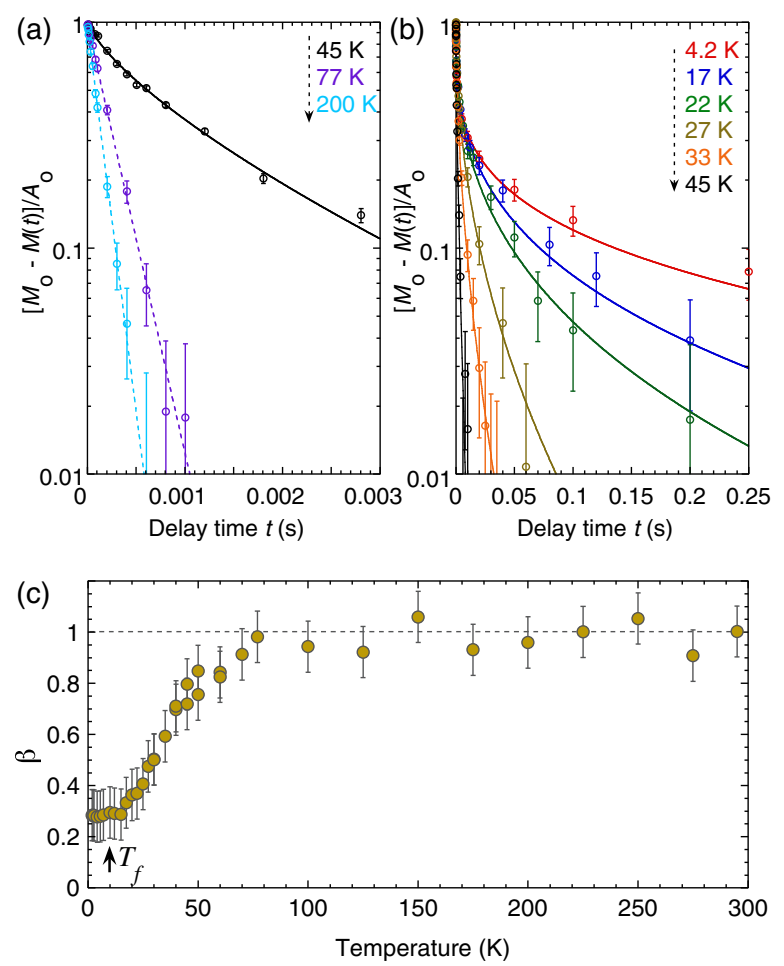

FIG. 13. (a),(b) The ${ }^{63} \mathrm{Cu}$ NQR $T_{1}$ recovery curves observed at representative temperatures for the $\mathrm{Cu}\left(\mathrm{I}_{1}\right)$ sites, presented in the form of $\left[M_{o}-M(t)\right] / A$. Dashed straight lines are the best exponential fit with Eq. (A1), while the solid curves are the best phenomenological stretched exponential fit with Eq. (A2). (c) The stretched exponent $\beta$ obtained from the fit with Eq. (A2) choosing $\beta$ as a free parameter.

by the $P\left(1 / T_{1}\right)$ results in Fig. 8 based on the inverse Laplace transform of $M(t)$. That is, the center of gravity of the distribution function $P\left(1 / T_{1}\right)$ agrees fairly well with $1 / T_{1, \mathrm{str}}$.

Our goal, however, is not merely to find the sample averaged behavior. A better approach to capture the intrinsic slow component $1 / T_{1 \text {,slow }}$ is the more traditional two-component fit of $M(t)$ :

$M(t)=M_{o}-A_{1} \exp \left(-3 t / T_{1, \text { slow }}\right)-A_{2} \exp \left(-\left[3 t / T_{1, \text { fast }}\right]^{\beta}\right)$.

Here, $M_{o}, A_{1}$, the intrinsic slow component $1 / T_{1, \text { slow }}, A_{2}$, and the defect-induced fast component $1 / T_{1 \text {,fast }}$ are the fitting parameters. We also introduced the phenomenological stretched exponent $\beta$ in the second term to improve the fit [the estimated values of $\beta$ from Eq. (A3) are similar to those from Eq. (A2)]. It is worth noting that an analogous two-component fit was previously applied successfully to diluted antiferromagnets $\mathrm{Mn}_{1-x} \mathrm{Zn}_{x} \mathrm{~F}_{2}$ [43]. (The sitedilution effect caused by nonmagnetic $\mathrm{Zn}^{2+}$ ions in $\mathrm{Mn}_{1-x} \mathrm{Zn}_{x} \mathrm{~F}_{2}$ results in the distributed slow component of $1 / T_{1}$ in the vicinity of $\mathrm{Zn}^{2+}$ ions, while the fast intrinsic component obeys the single exponential form.)
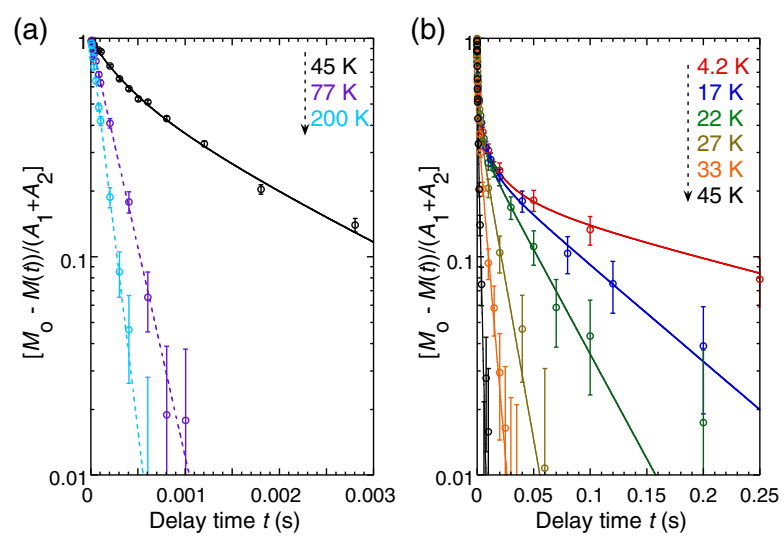

FIG. 14. (a),(b) Solid curves represent the two-component fit with Eq. (A3) above (a) and below (b) $45 \mathrm{~K}$, presented in the form of $\left[M_{o}-M(t)\right] /\left(A_{1}+A_{2}\right)$. Notice that after the initial

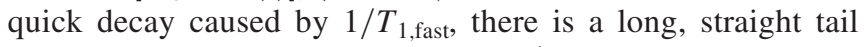
associated with the slow component $1 / T_{1, \text { slow }}$. Dashed straight lines through the 77 and $200 \mathrm{~K}$ data points are the best exponential fit with Eq. (A1).

Aside from the precedent, a concrete justification of the two-component fit in the present case comes from the fact that the inverse Laplace transform of $M(t)$ indeed shows the presence of two peaks in a wide temperature range below $\sim 60 \mathrm{~K}$ down to $T_{f}$, as shown in Fig. 8 . Figures 14(a) and 14(b) show the normalized recovery curves $\left[M_{o}-M(t)\right] /\left(A_{1}+A_{2}\right)$ plotted in a semilogarithmic scale; the distinct slow component $1 / T_{1 \text {,slow }}$ manifests itself as a straight segment after the initial quick decay due to $1 / T_{1, \text { fast. }}$. The $1 / T_{1}$ results below $\sim 60 \mathrm{~K}$ plotted in Figs. 5(b), 6(a), 7(a), and 7(b) with filled symbols are $1 / T_{1, \text { slow }}$ based on the fit with Eq. (A3). The open symbols in Figs. 5(b) and 7 (a) represent $1 / T_{1, \text { str }}$ from the fit with Eq. (A2) or $1 / T_{1, \text { fast }}$ from the fit with Eq. (A3).

\section{APPENDIX B: ${ }^{63,65} \mathrm{Cu}$ NMR MEASUREMENTS IN HIGH MAGNETIC FIELD $B_{\text {ext }}$}

\section{Field sweep ${ }^{63,65} \mathrm{Cu}$ NMR line shapes}

In Fig. 15, we compare the ${ }^{63,65} \mathrm{Cu}$ NMR line shapes measured at $90.307 \mathrm{MHz}$ for uniaxially aligned and unaligned powder samples at $10 \mathrm{~K}$ while sweeping the external magnetic field. For the purpose of determining the NMR Knight shift accurately, we measured only the $I_{z}=-1 / 2$ to $+1 / 2$ central peak region in a fixed magnetic field of $B_{\mathrm{ext}}=9 \mathrm{~T}$ while sweeping the frequency, as shown in Figs. 9(a)-9(c).

The line shape for the unaligned powder sample in Fig. 15(c) exhibits a typical powder pattern with a double horn structure arising from the $I_{z}=-1 / 2$ to $+1 / 2$ central transition of the ${ }^{63} \mathrm{Cu}$ and ${ }^{65} \mathrm{Cu}$ isotopes, as identified by filled green arrows. The horn at the lower-field side originates from nuclear spins in the grains whose primary principal axis of the electric field gradient tensor (i.e., the crystal $c$ axis) forms a right angle $\theta \sim 90^{\circ}$ with the external 


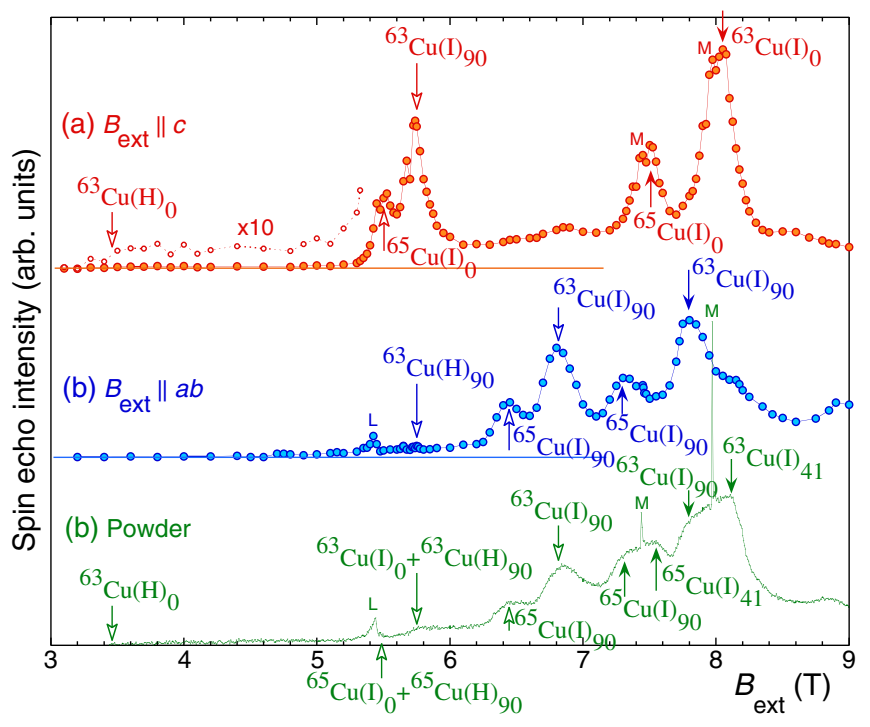

FIG. 15. Field sweep ${ }^{63,65} \mathrm{Cu}$ NMR line shapes observed at $10 \mathrm{~K}$ at a fixed frequency of $90.307 \mathrm{MHz}$ for (a),(b) a uniaxially aligned powder and (c) an unaligned powder. Key features associated with ${ }^{63} \mathrm{Cu}\left({ }^{65} \mathrm{Cu}\right)$ isotope are marked with downward (upward) arrows. The subscripted numbers 0, 41, and 90 denote the angle $\theta$ between $B_{\text {ext }}$ and the crystal $c$ axis. Filled and open arrows distinguish the features arising from the $I_{z}=+1 / 2$ to $-1 / 2$ central transition and $I_{z}= \pm 1 / 2$ to $\pm 3 / 2$ satellite transition. Peaks designated with the letter $M$ (or $L$ ) are background signals from ${ }^{63} \mathrm{Cu}$ and ${ }^{65} \mathrm{Cu}$ metal parts (or ${ }^{7} \mathrm{Li}$ ) in the sample environment.

magnetic field, whereas the horn at the higher-field side has $\theta \sim 41^{\circ}$. (Their relative locations are reversed in the frequency sweep line shapes in Fig. 9.) These structures are smeared by magnetic line broadening at $10 \mathrm{~K}$ caused by defect spins.

When we apply $B_{\text {ext }}$ along the $a b$ plane of the aligned powder sample, the $\theta \sim 90^{\circ}$ peak is enhanced, as seen in both Figs. 15(b) and 9(c). On the other hand, when we apply $B_{\text {ext }}$ along the aligned $c$ axis, a new peak emerges in the middle of the $\theta \sim 90^{\circ}$ and $\theta \sim 41^{\circ}$ peaks, as seen in Figs. 15(a) and Fig. 9(b); this is because the effects of the nuclear quadrupole interaction on the NMR frequency shift $\Delta \nu_{Q}^{(2)}$ vanishes for both $\mathrm{Cu}(\mathrm{I})$ and $\mathrm{Cu}(\mathrm{H})$ along $\theta \sim 0^{\circ}$ due to their negligibly small asymmetry of the EFG tensor. Since the NMR properties of the $\mathrm{Cu}\left(\mathrm{I}_{1}\right)$ and $\mathrm{Cu}\left(\mathrm{I}_{2}\right)$ sites are very similar, we were unable to clearly resolve their broad NMR lines.

At $10 \mathrm{~K}$, the $\mathrm{Cu}(\mathrm{H})$ central peaks are hidden by stronger signals arising from the $\mathrm{Cu}\left(\mathrm{I}_{1}\right)$ and $\mathrm{Cu}\left(\mathrm{I}_{2}\right)$ sites, because the quadrupole effects are very strong at $\mathrm{Cu}(\mathrm{H})$ sites and the NMR line is broadened by defect spins. Notice, however, that the ${ }^{63,65} \mathrm{Cu}$ NMR signal in Fig. 15 extends below the lower bound expected for the $I_{z}= \pm 1 / 2$ to $\pm 3 / 2$ satellite transitions of $\mathrm{Cu}(\mathrm{I})$ sites, and diminishes instead at the edge near $B_{\text {ext }} \sim 3.4 \mathrm{~T}$ as expected from ${ }^{63} \nu_{Q} \sim 52 \mathrm{MHz}$ at ${ }^{63} \mathrm{Cu}(\mathrm{H})$ sites.

\section{Magnetic field effect on $1 / T_{1}$}

We tested the potential effect of external magnetic field $B_{\text {ext }}$ on $\operatorname{Ir}$ spin dynamics by measuring $1 / T_{1}$ for the aligned powder sample in $B_{\text {ext }}=9 \mathrm{~T}$, using the $\theta \sim$ $90^{\circ} I_{z}= \pm 1 / 2$ to $\pm 3 / 2$ lower satellite transition for the ${ }^{63} \mathrm{Cu}(\mathrm{I})$ sites. The standard recovery function in this case takes the form of $[36,37]$

$$
\begin{aligned}
M(t)= & M_{o}-A\left[0.4 \exp \left(-6 t / T_{1}\right)\right. \\
& \left.+0.5 \exp \left(-3 t / T_{1}\right)+0.1 \exp \left(-t / T_{1}\right)\right] .
\end{aligned}
$$

In analogy with the two-component fitting function Eq. (A3) for NQR, we used the two-component fit below $\sim 60 \mathrm{~K}$ for the satellite transition as well,

$$
\begin{aligned}
M(t)= & M_{o}-A_{1}\left\{0.4 \exp \left[-\left(6 t / T_{1}\right)\right]\right. \\
& \left.+0.5 \exp \left[-\left(3 t / T_{1}\right)\right]+0.1 \exp [-(t / T 1)]\right\} \\
& -A_{2}\left\{0.4 \exp \left[-\left(6 t / T_{1, \text { fast }}\right)^{\beta}\right]\right. \\
& +0.5 \exp \left[-\left(3 t / T_{1, \text { fast }}\right)^{\beta}\right] \\
& \left.+0.1 \exp \left[-\left(t / T_{1, \text { fast }}\right)^{\beta}\right]\right\},
\end{aligned}
$$

as shown in Fig. 16. The high-field NMR $1 / T_{1}$ results presented with $\times$ symbols in Figs. 6(a) and 7(a) are nothing but $1 / T_{1 \text {,slow }}$ from the first term in Eq. (B2), and the temperature dependence is nearly identical with the NQR results. $1 / T_{1, \text { fast }}$ and $\beta$ estimated from Eq. (B2) are also similar to the NQR results.

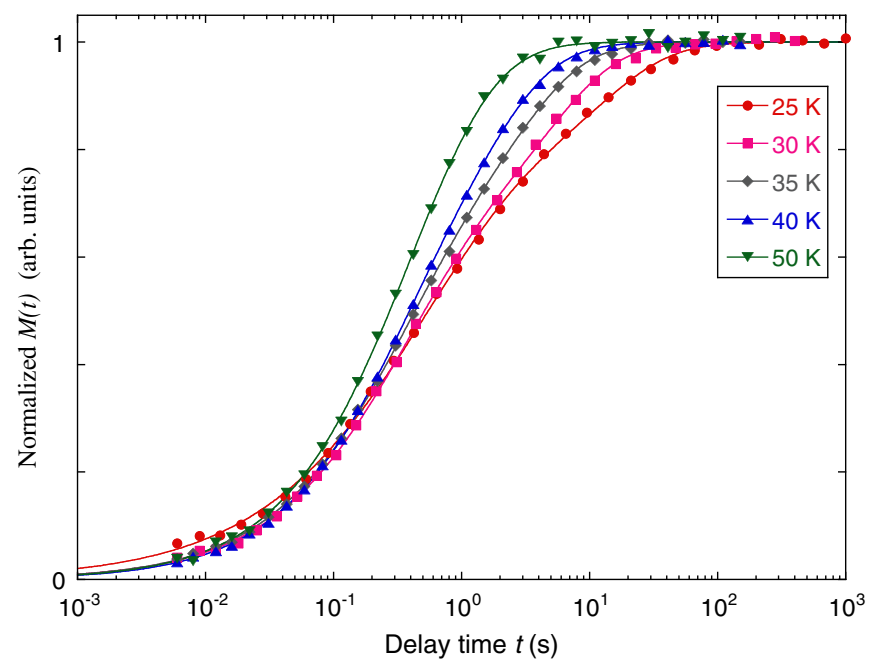

FIG. 16. Normalized $T_{1}$ recovery curves $M(t)$ measured in $B_{\text {ext }}=9 \mathrm{~T}$ at selected temperatures for the $I_{z}= \pm 1 / 2$ to $\pm 3 / 2$ lower satellite transition of ${ }^{63} \mathrm{Cu}(\mathrm{I})$ sites. Solid curves represent the two-component fit with Eq. (B2). 


\section{APPENDIX C: ${ }^{23} \mathrm{Na}$ NMR MEASUREMENTS IN $\mathrm{Na}_{2} \mathrm{IrO}_{3}$}

In Fig. 17, we summarize ${ }^{23} \mathrm{Na}$ (nuclear spin $I=3 / 2$ ) powder NMR line shapes measured in $B_{\text {ext }}=9 \mathrm{~T}$. At $295 \mathrm{~K}$, we observed two central peaks from the nuclear spin $I_{z}=+1 / 2$ to $-1 / 2$ transition. We assign the main peak centered at $\sim 101.37 \mathrm{MHz}$ to the interlayer ${ }^{23} \mathrm{Na}(\mathrm{I})$ sites. The smaller side peak at higher frequency $\sim 101.46 \mathrm{MHz}$ with larger Knight shift should be attributed to the less abundant sodium site within the honeycomb plane, referred to as ${ }^{23} \mathrm{Na}(\mathrm{H})$. We were also able to resolve shoulders from $I_{z}= \pm 3 / 2$ to $\pm 1 / 2$ satellite transitions at $\sim 102.1$ and $\sim 100.8 \mathrm{MHz}$. An additional small peak or shoulder at $\sim 101.3 \mathrm{MHz}$, marked with $*$, is at the nonshifted frequency, and should be attributed to a small amount of nonmagnetic impurity phase. The line shape broadens dramatically below $T_{N} \sim 16.5 \mathrm{~K}$ due to the emergence of static hyperfine magnetic fields from the ordered Ir moments.

We also measured $1 / T_{1}$ at the relatively isolated ${ }^{23} \mathrm{Na}(\mathrm{I})$ central peak. We summarize the recovery curves $M(t)$ in Fig. 18 and the temperature dependence of $1 / T_{1}^{\mathrm{Na}}$ in Fig. 6(b). Despite the superposition of NMR signals arising from the satellite transitions of the ${ }^{23} \mathrm{Na}(\mathrm{I})$ sites as well as ${ }^{23} \mathrm{Na}(\mathrm{H})$ sites, the fit of the recovery curve $M(t)$ with the standard form for the central transition [36,37],

$$
\begin{aligned}
M(t)= & M_{o}-A\left\{0.9 \exp \left[-(6 t / T 1)^{\beta}\right]\right. \\
& \left.+0.1 \exp \left[-(t / T 1)^{\beta}\right]\right\}
\end{aligned}
$$

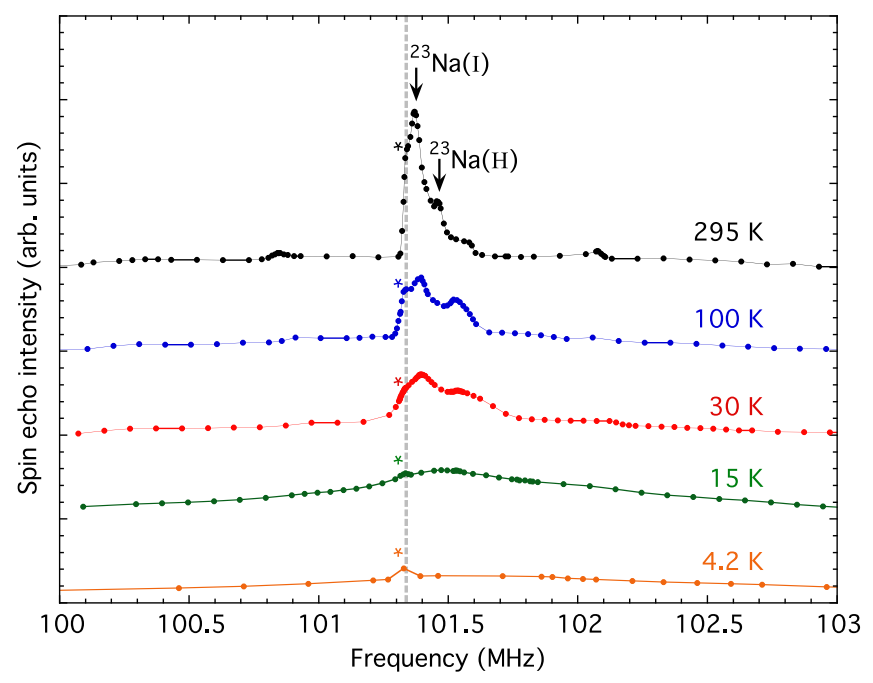

FIG. 17. ${ }^{23} \mathrm{Na}$ powder NMR line shapes observed at $B_{\text {ext }}=$ $9 \mathrm{~T}$ for $\mathrm{Na}_{2} \mathrm{IrO}_{3}$. The gray dashed vertical line marks the unshifted ${ }^{23} \mathrm{Na}$ frequency ${ }^{23} \nu_{0}$. The hump marked with $*$ is an unshifted ${ }^{23} \mathrm{Na}$ signal from a small amount of magnetically inert impurity phase.

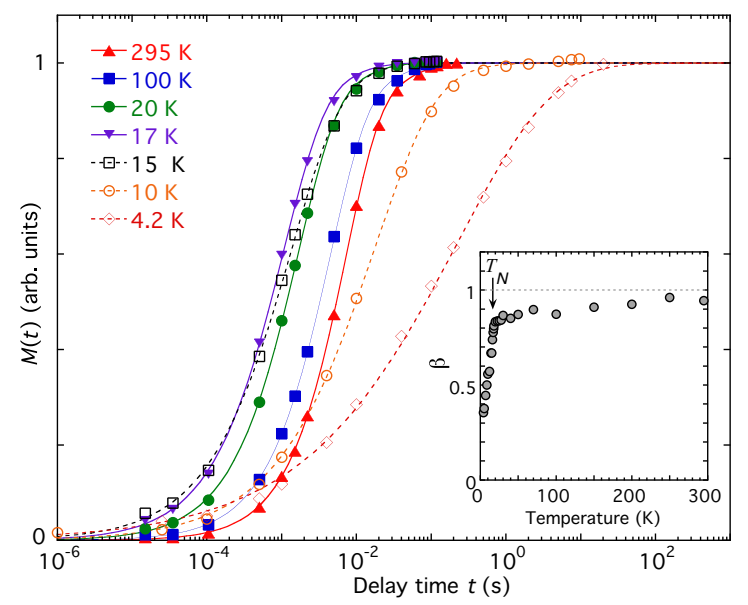

FIG. 18. $T_{1}$ recovery curves for the nuclear spin $I_{z}=+1 / 2$ to $-1 / 2$ central transition of the interlayer ${ }^{23} \mathrm{Na}(\mathrm{I})$ sites of $\mathrm{Na}_{2} \mathrm{IrO}_{3}$ at select temperatures. Solid and dashed curves represent the fit with Eq. (C1) with the stretched exponent $\beta$ above and below $T_{N}$, respectively. The magnitude of $\beta$ is close to 1 above $T_{N}$, as shown in the inset.

was good with fixed $\beta=1$ down to $\sim 30 \mathrm{~K}$. To improve the fit below $T_{N}$, we introduced the stretched exponent $\beta$ as summarized in the inset of Fig. 18. Since the value of $\beta$ remains close to $\sim 1$ above $T_{N}$, the qualitatively important aspects of $1 / T_{1}$ results in Fig. 6(b) do not depend significantly on the details of the fit, with or without $\beta$.

[1] P. A. Lee, An End to the Drought of Quantum Spin Liquids, Science 321, 1306 (2008).

[2] L. Balents, Spin Liquids in Frustrated Magnets, Nature (London) 464, 199 (2010).

[3] J. G. Rau, E. K. H. Lee, and H. Y. Kee, Spin-Orbit Physics Giving Rise to Novel Phases in Correlated Systems: Iridates and Related Materials, Annu. Rev. Condens. Matter Phys. 7, 195 (2016).

[4] T. Imai and Y. S. Lee, Do Quantum Spin Liquids Exist?, Phys. Today 69, No. 8, 30 (2016).

[5] A. Kitaev, Anyons in an Exactly Solved Model and Beyond, Ann. Phys. (Amsterdam) 321, 2 (2006).

[6] X. Liu, T. Berlijn, W.-G. Yin, W. Ku, A. Tsvelik, Y.-J. Kim, H. Gretarsson, Y. Singh, P. Gegenwart, and J. P. Hill, LongRange Magnetic Ordering in $\mathrm{Na}_{2} \mathrm{IrO}_{3}$, Phys. Rev. B 83, 220403(R) (2011).

[7] S. K. Choi, R. Coldea, A. N. Kolmogorov, T. Lancaster, I. I. Mazin, S. J. Blundell, P. G. Radaelli, Y. Singh, P. Gegenwart, K. R. Choi, S.-W. Cheong, P. J. Baker, C. Stock, and J. Taylor, Spin Waves and Revised Crystal Structure of Honeycomb Iridate $\mathrm{Na}_{2} \mathrm{IrO}_{3}$, Phys. Rev. Lett. 108, 127204 (2012).

[8] J. Knolle, D. L. Kovrizhin, J. T. Chalker, and R. Moessner, Dynamics of Fractionalization in Quantum Spin Liquids, Phys. Rev. B 92, 115127 (2015).

[9] J. Yoshitake, J. Nasu, and Y. Motome, Fractional Spin Fluctuations as a Precursor of Quantum Spin Liquids: 
Majorana Dynamical Mean-Field Study for the Kitaev model, Phys. Rev. Lett. 117, 157203 (2016).

[10] J. Yoshitake, J. Nasu, Y. Kato, and Y. Motome, Majorana Dynamical Mean-Field Study of Spin Dynamics at Finite Temperatures in the Honeycomb Kitaev Model, Phys. Rev. B 96, 024438 (2017).

[11] J. Yoshitake, J. Nasu, and Y. Motome, Temperature Evolution of Spin Dynamics in Two- and Three-Dimensional Kitaev Models: Influence of Fluctuating $Z_{2}$ Flux, Phys. Rev. B 96, 064433 (2017).

[12] Y. Singh and P. Gegenwart, Antiferromagnetic Mott Insulating State in Single Crystals of the Honeycomb Lattice Material $\mathrm{Na}_{2} \mathrm{IrO}_{3}$, Phys. Rev. B 82, 064412 (2010).

[13] P. Khuntia, S. Manni, F. R. Foronda, T. Lancaster, S. J. Blundell, P. Gegenwart, and M. Baenitz, Local Magnetism and Spin Dynamics of the Frustrated Honeycomb Rhodate $\mathrm{Li}_{2} \mathrm{RhO}_{3}$, Phys. Rev. B 96, 094432 (2017).

[14] L. J. Sandilands, Y. Tian, K. W. Plumb, Y.-J. Kim, and K. S. Burch, Scattering Continuum and Possible Fractionalized Excitations in $\alpha-\mathrm{RuCl}_{3}$, Phys. Rev. Lett. 114, 147201 (2015).

[15] K. Kitagawa, T. Takayama, Y. Matsumoto, A. Kato, R. Takano, Y. Kishimoto, S. Bette, G. Dinnerbier, G. Jackeli, and H. Takagi, A Spin-Orbit-Entangled Quantum Liquid on a Honeycomb Lattice, Nature (London) 554, 341 (2018).

[16] G. Jackeli and G. Khaliullin, Mott Insulators in the Strong Spin-Orbit Coupling Limit: From Heisenberg to a Quantum Compass and Kitaev Models, Phys. Rev. Lett. 102, 017205 (2009).

[17] J. Chaloupka, G. Jackeli, and G. Khaliullin, KitaevHeisenberg Model on a Honeycomb Lattice: Possible Exotic Phases in Iridium Oxides $\mathrm{A}_{2} \mathrm{IrO}_{3}$, Phys. Rev. Lett. 105, 027204 (2010).

[18] J. Chaloupka, G. Jackeli, and G. Khaliullin, Zigzag Magnetic Order in the Iridium Oxide $\mathrm{Na}_{2} \mathrm{IrO}_{3}$, Phys. Rev. Lett. 110, 097204 (2013).

[19] A. Banerjee, C. Bridges, J. Yan, A. Aczel, L. Li, M. Stone, G. Granroth, M. Lumsden, Y. Yiu, J. Knolle, S. Bhattacharjee, D. Kovrizhin, R. Moessner, D. Tennant, D. Mandrus, and S. Nagler, Proximate Kitaev Quantum Spin Liquid Behaviour in a Honeycomb Magnet, Nat. Mater. 15 (2016).

[20] A. Banerjee, J. Yan, J. Knolle, C. A. Bridges, M. B. Stone, M. D. Lumsden, D. G. Mandrus, D. A. Tennant, R. Moessner, and S.E. Nagler, Neutron Scattering in the Proximate Quantum Spin Liquid $\alpha$-RuCl${ }_{3}$, Science 356, 1055 (2017).

[21] A. Banerjee, P. Lampen-Kelley, J. Knolle, C. Balz, A. A. Aczel, B. Winn, Y. Liu, D. Pajerowski, J. Yan, C. A. Bridges, A. T. Savici, B. C. Chakoumakos, M. D. Lumsden, D. A. Tennant, R. Moessner, D. G. Mandrus, and S. E. Nagler, Excitations in the Field-Induced Quantum Spin Liquid State of $\alpha-\mathrm{RuCl}_{3}$, npj Quantum Mater. 3, 8 (2018).

[22] M. Abramchuk, C. Ozsoy-Keskinbora, J. W. Krizan, K. R. Metz, D. C. Bell, and F. Tafti, $\mathrm{Cu}_{2} \mathrm{IrO}_{3}$ : A New Magnetically Frustrated Honeycomb Iridate, J. Am. Chem. Soc. 139, 15371 (2017).

[23] Y. S. Choi, C. H. Lee, S. Lee, S. Yoon, W.-J. Lee, J. Park, A. Ali, Y. Singh, J.-C. Orain, G. Kim, J.-S. Rhyee, W.-T. Chen, F. Chou, and K.-Y. Choi, Exotic Low-Energy Excitations Emergent in the Random Kitaev Magnet $\mathrm{Cu}_{2} \mathrm{IrO}_{3}$, Phys. Rev. Lett. 122, 167202 (2019).
[24] S. M. Winter, A. A. Tsirlin, M. Daghofer, J. van der Brink, Y. Singh, P. Gegenwart, and R. Valenti, Models and Materials for Generalized Kitaev Magnetism, J. Phys. Condsen. Matter 29, 493002 (2017).

[25] E. Kenney, C. U. Segre, W. Lafargue-Dit-Hauret et al., Competition between Static and Dynamic Magnetism in the Kitaev Spin Liquid Material $\mathrm{Cu}_{2} \mathrm{IrO}_{3}$, arXiv:1811 .00565 .

[26] I. Kimchi, J. P. Sheckelton, T. M. McQueen, and P. Lee, Scaling and Data Collapse from Local Moments in Frustrated Disordered Quantum Spin Systems, Nat. Commun. 9, 4367 (2018).

[27] S. Manni, Y. Tokiwa, and P. Gegenwart, Effect of Nonmagnetic Dilution in the Honeycomb-Lattice Iridates $\mathrm{Na}_{2} \mathrm{IrO}_{3}$ and $\mathrm{Li}_{2} \mathrm{IrO}_{3}$, Phys. Rev. B 89, 241102(R) (2014).

[28] S. Manni, S. Choi, I. I. Mazin, R. Coldea, M. Altmeyer, H. O. Jeschke, R. Valentí, and P. Gegenwart, Effect of Isoelectronic Doping on the Honeycomb-Lattice Iridate $A_{2} \mathrm{IrO}_{3}$, Phys. Rev. B 89, 245113 (2014).

[29] D. C. Wallace, C. M. Brown, and T. M. McQueen, Evolution of Magnetism in $\mathrm{Na}_{3-d}\left(\mathrm{Na}_{1-x} \mathrm{Mg}_{x}\right) \mathrm{Ir}_{2} \mathrm{O}_{6}$ Series of Honeycomb Iridates, J. Solid State Chem. 224, 28 (2015).

[30] J. Knolle, R. Moessner, and N. B. Perkins, Bond-Disordered Spin Liquid and the Honeycomb Iridate $\mathrm{H}_{3} \mathrm{LiIr}_{2} \mathrm{O}_{6}$ : Abundant Low-Energy Density of States from Random Majorana Hopping, Phys. Rev. Lett. 122, 047202 (2019).

[31] F. Mila and T. M. Rice, Analysis of Magnetic Resonance Experiments in $\mathrm{YBa}_{2} \mathrm{Cu}_{3} \mathrm{O}_{7}$, Physica (Amsterdam) 157C, 561 (1989).

[32] T. Imai, E. A. Nytko, B. M. Bartlett, M. P. Shores, and D. G. Nocera, ${ }^{63} \mathrm{Cu},{ }^{35} \mathrm{Cl}$, and ${ }^{1} \mathrm{H} N M R$ in the $S=\frac{1}{2}$ Kagome Lattice $\mathrm{ZnCu}_{3}(\mathrm{OH})_{6} \mathrm{Cl}_{2}$, Phys. Rev. Lett. 100, 077203 (2008).

[33] T. Imai, M. Fu, T. H. Han, and Y. S. Lee, Local Spin Susceptibility of the $S=\frac{1}{2}$ Kagome Lattice in $\mathrm{ZnCu}_{3}(\mathrm{OD})_{6} \mathrm{Cl}_{2}$, Phys. Rev. B 84, 020411(R) (2011).

[34] M. Fu, T. Imai, T. H. Han, and Y. S. Lee, Evidence for a Gapped Spin-Liquid State in a Kagome Anitiferromagnet, Science 350, 655 (2015).

[35] M. Abramchuk, O. I. Lebedev, O. Hellman, F. Bahrami, N. E. Mordvinova, J. W. Krizan, K. R. Metz, D. Broido, and F. Tafti, Crystal Chemistry and Phonon Heat Capacity in Quaternary Honeycomb Delafossites: $\mathrm{Cu}\left[\mathrm{Li}_{1 / 3} \mathrm{Sn}_{2 / 3}\right] \mathrm{O}_{2}$ and $\mathrm{Cu}\left[\mathrm{Na}_{1 / 3} \mathrm{Sn}_{2 / 3}\right] \mathrm{O}_{2}$, Inorg. Chem. 57, 12709 (2018).

[36] E. R. Andrew and D. P. Tunstall, Spin-Lattice Relaxation in Imperfect Cubic Crystals and in Non-Cubic Crystals, Proc. R. Soc. 78, 1 (1961).

[37] A. Narath, Nuclear Spin Lattice Relaxation in Hexagonal Transition Metals: Titanium, Phys. Rev. 162, 320 (1967).

[38] M. Takigawa, P. C. Hammel, R. H. Heffner, Z. Fisk, J. L. Smith, and R. B. Schwarz, Anisotropic Cu Knight Shift and Magnetic Susceptibility in the Normal State of $\mathrm{YBa}_{2} \mathrm{Cu}_{3} \mathrm{O}_{7}$, Phys. Rev. B 39, 300 (1989).

[39] A. J. Millis, H. Monien, and D. Pines, Phenomenological Model of Nuclear Relaxation in the Normal State of $\mathrm{YBa}_{2} \mathrm{Cu}_{3} \mathrm{O}_{7}$, Phys. Rev. B 42, 167 (1990).

[40] D. C. Johnston, S.-H. Baek, X. Zong, F. Borsa, J. Schmalian, and S. Kondo, Dynamics of Magnetic Defects in Heavy Fermion $\mathrm{LiV}_{2} \mathrm{O}_{4}$ from Stretched Exponential ${ }^{7} \mathrm{Li} N M R$ Relaxation, Phys. Rev. Lett. 95, 176408 (2005). 
[41] P. M. Singer, D. Asthagiri, W. G. Chapman, and G. J. Hirasaki, NMR Spin-Rotation Relaxation and Diffusion of Methane, J. Chem. Phys. 148, 204504 (2018).

[42] P. Thayamballi and D. Hone, Nuclear Relaxation in a Randomly Diluted Heisenberg Paramagnet, Phys. Rev. B 21, 1766 (1980).

[43] M. Itoh, H. Yasuoka, A. R. King, and V. Jaccarino, Decay of the Nuclear Magnetization in the Randomly Diluted Antiferromagnets $\mathrm{Fe}_{x} \mathrm{Zn}_{1-x} \mathrm{~F}_{2}$ and $\mathrm{Mn}_{x} \mathrm{Zn}_{1-x} \mathrm{~F}_{2}$, J. Phys. Soc. Jpn. 55, 964 (1986).

[44] L. Venkataramanan, Y. Q. Song, and M. D. Hürlimann, Solving Fredholm Integrals of the First Kind with Tensor Product Structure in 2 and 2.5 Dimensions, IEEE Trans. Signal Process. 50, 1017 (2002).

[45] Y. Q. Song, L. Venkataramanan, M. D. Hürlimann, M. Flaum, P. Frulla, and C. Straley, $T_{1}-T_{2}$ Correlation Spectra Obtained Using a Fast Two-Dimensional Laplace Inversion, J. Magn. Reson. 154, 261 (2002).

[46] G. Simutis, N. Barbero, K. Rolfs, P. Leroy-Calatayud, K. Mehlawat, R. Khasanov, H. Luetkens, E. Pomjakushina, Y. Singh, H.-R. Ott, J. Mesot, A. Amato, and T. Shiroka, Chemical and Hydrostatic-Pressure Effects on the Kitaev Honeycomb Material $\mathrm{Na}_{2} \mathrm{IrO}_{3}$, Phys. Rev. B 98, 104421 (2018).

[47] Y. Itoh, T. Imai, T. Shimizu, T. Tsuda, H. Yasuoka, and Y. Ueda, Nuclear Quadrupole Resonance of $\mathrm{Cu}$ in the Paramagnetic State of CuO, J. Phys. Soc. Jpn. 59, 1143 (1990).

[48] D. Beeman and P. Pincus, Nuclear Spin-Lattice Relaxation in Magnetic Insulators, Phys. Rev. 166, 359 (1968).

[49] T. Tsuda, T. Ohno, and H. Yasuoka, Spin-Lattice Relaxation of ${ }^{63} \mathrm{Cu}$ in High- $T_{c}$ Related Antiferromagnetic Cu Oxides, J. Phys. Soc. Jpn. 61, 2109 (1992).

[50] S.-H. Baek, S.-H. Do, K.-Y. Choi, Y. S. Kwon, A. U. B. Wolter, S. Nishimoto, J. van den Brink, and B. Büchner, Evidence for a Field-Induced Quantum Spin Liquid in $\alpha-\mathrm{RuCl}_{3}$, Phys. Rev. Lett. 119, 037201 (2017).
[51] J. Zheng, K. Ran, T. Li, J. Wang, P. Wang, B. Liu, Z.-X. Liu, B. Normand, J. Wen, and W. Yu, Gapless Spin Excitations in the Field-Induced Quantum Spin Liquid Phase of $\alpha$ - $\mathrm{RuCl}_{3}$, Phys. Rev. Lett. 119, 227208 (2017).

[52] N. Jansa, A. Zorko, M. Gomilsek, M. Pregelj, K. W. Kramer, D. Biner, A. Biffin, C. Ruegg, and M. Klanjsek, Observation of Two Types of Fractional Excitation in the Kitaev Honeycomb Magnet, Nat. Phys. 14, 786 (2018).

[53] Y. Kasahara, T. Ohnishi, Y. Mizukami, O. Tanaka, S. Ma, K. Sugii, N. Kurita, H. Tanaka, J. Nasu, Y. Motome, T. Shibauchi, and Y. Matsuda, Majorana Quantization and Half-Integer Thermal Quantum Hall Effect in a Kitaev Spin Liquid, Nature (London) 559, 227 (2018).

[54] L. E. Chern, R. Kaneko, H.-Y. Lee, and Y. B. Kim, Magnetic Field Induced Competing Phases in Spin-Orbital Entangled Kitaev Magnets, arXiv:1905.11408.

[55] A. W. Kinross, M. Fu, T. J. Munsie, H. A. Dabkowska, G. M. Luke, S. Sachdev, and T. Imai, Evolution of Quantum Fluctuations Near the Quantum Critical Point of the Transverse Field Ising Chain System $\mathrm{CoNb}_{2} \mathrm{O}_{6}$, Phys. Rev. X 4, 031008 (2014).

[56] M. Azuma, Z. Hiroi, M. Takano, K. Ishida, and Y. Kitaoka, Observation of a Spin Gap in $\mathrm{SrCu}_{2} \mathrm{O}_{3}$ Comprising Spin-1/2 Quasi-1D Two-Leg Ladders, Phys. Rev. Lett. 73, 3463 (1994).

[57] T. Imai, K. R. Thurber, K. M. Shen, A. W. Hunt, and F. C. Chou, ${ }^{17} \mathrm{O}$ and ${ }^{63} \mathrm{Cu}$ NMR in Undoped and Hole Doped $\mathrm{Cu}_{2} \mathrm{O}_{3}$ Two-Leg Spin Ladder $\mathrm{A}_{14} \mathrm{Cu}_{24} \mathrm{O}_{41}\left(\mathrm{~A}_{14}=\mathrm{La}_{6} \mathrm{Ca}_{8}\right.$, $\mathrm{Sr}_{14}, \mathrm{Sr}_{11} \mathrm{Ca}_{3}$ ), Phys. Rev. Lett. 81, 220 (1998).

[58] K. R. Thurber, A. W. Hunt, T. Imai, F. C. Chou, and Y. S. Lee, ${ }^{17} \mathrm{O}$ NMR Study of Undoped and Lightly Hole Doped $\mathrm{CuO}_{2}$ Planes, Phys. Rev. Lett. 79, 171 (1997).

[59] M. Greven, R. J. Birgeneau, Y. Endoh, M. A. Kastner, M. Matsuda, and G. Shirane, Neutron Scattering Study of the Two-Dimensional Spin $S=1 / 2$ Square-Lattice Heisenberg Antiferromagnet $\mathrm{Sr}_{2} \mathrm{CuO}_{2} \mathrm{Cl}_{2}$, Z. Phys. B 96, 465 (1995). 